\title{
Copyright
}

by

Michael Edward Cholette

2009 
The Thesis committe for Michael Edward Cholette certifies that this is the approved version of the following Thesis:

\section{Precedent-Free Fault Isolation in a Diesel Engine EGR Valve System}

APPROVED BY

SUPERVISING COMMITTEE:

Dragan Djurdjanovic, Supervisor

Benito R. Fernández 


\title{
Precedent-Free Fault Isolation in a Diesel Engine EGR Valve System
}

\author{
by
}

\section{Michael Edward Cholette, B.S.}

\author{
THESIS \\ Presented to the Faculty of the Graduate School of \\ The University of Texas at Austin \\ in Partial Fulfillment \\ of the Requirements \\ for the Degree of \\ MASTER OF SCIENCE IN ENGINEERING
}

THE UNIVERSITY OF TEXAS AT AUSTIN

December 2009 


\section{Acknowledgments}

First and foremost, I would like to thank my supervisor, Dragan Djurdjanovic, whose support, guidance and encouragement made this thesis possible. I would also like Dr. Benito Fernández for his review and valuable comments on my drafts as well as the valuable discussions we had when I was a student in his classes.

I would also like to thank my colleagues in the LIMES Lab. In particular, Marcus Musselman and Yibo Jiao, who were always willing to discuss ideas with me and Alex Bleakie who helped edit my thesis drafts.

Finally, I wish to thank my parents, Kathy and Gary Cholette, and the love of my life, Casey Crocket, for all their support from afar. 


\title{
Precedent-Free Fault Isolation in a Diesel Engine EGR Valve System
}

\author{
Michael Edward Cholette, M.S.E. \\ The University of Texas at Austin, 2009
}

Supervisor: Dragan Djurdjanovic

\begin{abstract}
An application of a recently introduced framework for isolating unprecedented faults for an automotive engine EGR valve system is presented. Using normal behavior data generated by a high fidelity engine simulation, the Growing Structure Multiple Model System (GSMMS) is used to construct models of normal behavior for EGR valve system and its various subsystems. Using the GSMMS models as a foundation, anomalous behavior of the entire system is then detected as statistically significant departures of the most recent modeling residuals from the modeling residuals during normal behavior. By reconnecting anomaly detectors to the constituent subsystems, the anomaly can be isolated without the need for prior training using faulty data. Furthermore, faults that were previously encountered (and modeled) are recognized using the same approach as the anomaly detectors.
\end{abstract}




\section{Table of Contents}

Acknowledgments iv

$\begin{array}{lll}\text { Abstract } & \text { v }\end{array}$

List of Tables viii

List of Figures $\quad$ ix

Chapter 1. Introduction 1

1.1 Motivation . . . . . . . . . . . . . . . . 1

1.2 Objectives and Challenges . . . . . . . . . . . . . 2

1.3 Organization of Thesis . . . . . . . . . . . . . 4

Chapter 2. State of the Art for Engine Air Path Diagnostics 5

2.1 Fault Detection . . . . . . . . . . . . . . 8

2.2 Fault Isolation . . . . . . . . . . . . . . . . . . . . 10

2.3 Fault Identification . . . . . . . . . . . . . . . . . . . 14

Chapter 3. GSMMS Based Anomaly Detection, Isolation and Identifica$\begin{array}{ll}\text { tion } & 17\end{array}$

3.1 The Growing Structure Multiple Model System (GSMMS) . . . . . 17

3.1.1 The GSMMS Modeling Approach . . . . . . . . . . . . . 18

3.1 .2 Training of the GSMMS . . . . . . . . . . . . 22

3.2 Analysis of Residuals for Fault Detection . . . . . . . . . . . . . . . 27

3.3 Anomaly Isolation Through Distributed Anomaly Detection . . . . . 30

3.4 GSMMS Based Fault Identification . . . . . . . . . . . . . . . . 31 
Chapter 4. A GSMMS Based Approach to Exhaust Gas Recirculation Diagnostics

4.1 Simulation Model and Fault Descriptions . . . . . . . . . . . . 35

4.2 EGR Diagnostic System Design . . . . . . . . . . . . . . . 40

4.3 Training the GSMMS Models . . . . . . . . . . . . . . 41

4.4 Fault Detection and Isolation $\ldots \ldots \ldots \ldots$

4.4 .1 EGR Valve Faults . . . . . . . . . . . . . . . . . 54

4.4 .2 Cooler Fault . . . . . . . . . . . . . . . . . . 54

4.4 .3 Controller Fault . . . . . . . . . . . . . . . 61

4.5 Fault Identification . . . . . . . . . . . . . . . . . . . . 65

$4.5 .1 \quad$ EGR Valve Faults $\ldots \ldots \ldots \ldots$

4.5 .2 EGR Cooler Faults . . . . . . . . . . . . . . . . . . . 65

4.5 .3 PI Controller Faults . . . . . . . . . . . . . . . 68

Chapter 5. Conclusions and Future Work 69

5.1 Conclusions . . . . . . . . . . . . . . . . . . . . . 69

5.2 Future Work . . . . . . . . . . . . . . . . . 72

5.2.1 Application to a Real Engine and Benchmarking . . . . . . 73

5.2.2 Controller Reconfiguration . . . . . . . . . . . . . 73

$\begin{array}{ll}\text { Bibliography } & 75\end{array}$

$\begin{array}{ll}\text { Vita } & 84\end{array}$ 


\section{List of Tables}

2.1 Isolation logic utilized in [1]. $r_{f}$ is a vector of residuals of the forward model. Similarly, $r_{i}$ is a vector of residuals of the inverse model. 11

4.1 Inputs for the various GSMMS models. . . . . . . . . . . . . . . 49

4.2 Model summary for the GSMMS models trained for anomaly detection. . . . . . . . . . . . . . 52

4.3 RMS errors of normal models computed over 661 seconds of a profile that was not used for training. . . . . . . . . . . . 52 


\section{List of Figures}

2.1 Model-based detection in automotive literature. . . . . . . . . . . 7

2.2 Engine NPERG scheme utilized in [1]. The forward and inverse NARMAX models are utilized to generate residual vectors $r_{f}$ and $r_{i}$ which are used in the fault isolation logic (see Table 2.1.) . . . . . 12

3.1 Voronoi Tessellation using 7 weight vectors $\boldsymbol{\xi}_{i}$. . . . . . . . . . . 20

3.2 Example of the topological distance calculation, $\operatorname{dis}(m, b)$. The the dashed contours enclose nodes that are the respective distances away from the Best Matching Unit (dis=0). Each of the nodes are the center of a model (Voronoi region). . . . . . . . . . . 23

3.3 Demonstration of growing of the SOM in the GSMMS. . . . . . . . 26

3.4 Flowchart for GSMMS training. . . . . . . . . . . . . . . 27

3.5 Illustration of the interpretation of the Confidence Value. . . . . . . 29

3.6 Illustration of fault isolation through distributed anomaly detection. 30

3.7 Depiction of the GSMMS based fault identification strategy. In the above example, $\mathrm{N}$ faults can be identified. . . . . . . . . . . . 32

4.1 Schematic of a generic EGR system. . . . . . . . . . . . . 34

4.2 Block diagram of the EGR valve system. . . . . . . . . . . . 35

4.3 Characteristic curves for the EGR valve. These curves are plots of $f_{2}$ in the valve model without (normal) and with four levels of obstruction $(10 \%, 20 \%, 30 \%, 40 \%$ and $50 \%)$ respectively. . . . . . 39

$4.4 K\left(\dot{m}_{\mathrm{EGR}}\right)$ for the EGR cooler for normal operation and four levels of fouling. As the fouling increases, the heat transfer coefficient, $\mathrm{K}$, decreases. . . . . . . . . . . . . . . . 40

4.5 Delay faults introduced into the EGR controller. . . . . . . . . . . 41

4.6 Simplified block diagram of the EGR system into a mass flow and temperature subsystem. . . . . . . . . . . . . . 42

4.7 Top level anomaly detection strategy for the EGR System. . . . . . 42

4.8 Fault Isolation in the EGR mass flow subsystem using distributed anomaly detection. . . . . . . . . . . . . . 43 
4.9 Driving profiles utilized for training and testing of the GSMMS models. . . . . . . . . . . . . . . . . 44

4.10 Example of a situation where a majority of the data is well represented by a single linear model, but a small region $(x>5.5)$ is modeled poorly. In the histogram, one sees that a result of the poor fit is a skewed residual distribution, leading to a significant number of points outside of the $4 \sigma$ limits. . . . . . . . . . . . . . . . 46

4.11 Using two models, the for $x>5.5$ is represented better and the residual histograms appear more Gaussian than with one model. This idea was the inspiration for the new node insertion criteria (Eq. 4.5). 47

4.12 Modification to GSMMS training procedure. . . . . . . . . . 48

4.13 Modeling Performance of the GSMMS for $\mathrm{AD}_{\text {cooler }}$ without a fault. The system was exercised with a standard driving profile (Japan 10-15) that was not utilized for training. . . . . . . . . . . . 50

4.14 Modeling Performance of the GSMMS for $\mathrm{AD}_{\text {valve }}$ without a fault. The system was exercised with a standard driving profile (Japan 10-15) that was not utilized for training. . . . . . . . . . . 51

4.15 Anomaly Detector hierarchy. ADs 2-5 are only monitored if the $\mathrm{AD}_{\text {mflow }} \mathrm{CV}$ is low. .................... 53

4.16 $\mathrm{CV}_{\text {global }}$ for the top level anomaly detectors. A valve anomaly introduced at 1322 seconds. Fault 1 is difficult to distinguish since since the $\mathrm{CV}$ change is small compared to the normal system. This is expected since $\mathrm{AD}_{\text {mflow }}$ is monitoring the closed-loop system and the controller is able to compensate for small faults. . . . . . . . . 55

4.17 Fault isolation using distributed anomaly detectors for a valve anomaly introduced at 1322 seconds. . . . . . . . . . . . . . . . 56

4.18 $\mathrm{CV}_{\text {global }}$ for the top level anomaly detectors a cooler anomaly introduced at 1322 seconds. As expected, the cooler AD CV is low. . . . 57

4.19 Mass flow subsystem CVs for a cooler anomaly. As expected, all of these CVs are near 1. . . . . . . . . . . . . . . 58

4.20 Extreme regional confidence values for $A D_{\text {cooler }}$ for Fault 1 . The $\mathrm{CV}$ s that drop the most (and least) during testing are shown. The remaining regional $\mathrm{CVs}$ are within these local extremes. . . . . . . 59

4.21 Local extreme confidence values for $\mathrm{AD}_{\text {cooler }}$ for Fault 2. The CVs that drop the most (and least) during testing are shown. The remaining regional CVs are within these local extremes. . . . . . . . . 60

4.22 Local extreme confidence values for $\mathrm{AD}_{\text {cooler }}$ for Fault 3. The CVs that drop the most (and least) during testing are shown. The remaining regional CVs are within these local extremes. . . . . . . . . . 60 
4.23 Local extreme confidence values for Fault $\mathrm{AD}_{\text {cooler }}$ for 4 . The CVs that drop the most (and least) during testing are shown. The remaining regional CVs are within these local extremes. . . . . . . . 61

4.24 $\mathrm{CV}_{\text {global }}$ for the top level anomaly detectors for a controller anomaly (time delay) introduced at 1322 seconds. . . . . . . . . . . 63

4.25 Fault isolation using distributed anomaly detectors for a controller anomaly (time delay) introduced at 1322 seconds. The $\mathrm{CV}$ for $\mathrm{AD}_{4}$ drops while other $\mathrm{CV}$ s remain high. It can also be seen that the amount of CV reduction is proportional to the delay. . . . . . . . . 64

4.26 Valve scaling factor ( $\gamma$ in Eq. 4.4) and $\mathrm{CV}_{\text {global }}$ for valve diagnosers for faults $2 \& 4$. The scaling factor determines the current behavior of the EGR Valve, where $\gamma=0.8$ is Fault 2 and $\gamma=0.5$ is Fault $4 . \quad$. 66

4.27 Cooler scaling factor and $\mathrm{CV}_{\text {global }}$ for cooler diagnosers for faults 2 $\& 4$. The scaling factor determines the current behavior of the EGR Cooler, where $\beta=0.8$ is Fault 2 and $\beta=0.5$ is Fault 4. . . . . . . 66

4.28 Regional CVs for Fault 4 diagnoser only. The regions shown here are clearly more sensitive than the global values in Fig. 4.27 and the regional CVs are high when Fault 4 is present, as expected. Note that the global CV for this diagnoser (4.27 is not nearly as sensitive as the local CVs shown. . . . . . . . . . . . . . . . 67

$4.2920 \mathrm{~ms}$ diagnoser $\mathrm{CV}$ for the PI controller in the presence of various delays. The CV during the $20 \mathrm{~ms}$ fault period is different from the $30 \mathrm{~ms}$ and normal periods, but the $10 \mathrm{~ms}$ fault period maintains a high CV. . . . . . . . . . . . . . . . . . . . 68 


\section{Chapter 1}

\section{Introduction}

\subsection{Motivation}

In order to meet increasingly demanding emission and fuel consumption requirements, automotive systems have become increasingly complex and sophisticated systems. In particular, the push to meet emission standards has given rise to numerous controlled subsystems that must be monitored in order to ensure performance. One such system is the Exhaust Gas Recirculation (EGR) system. EGR systems are used to lower combustion temperatures and reduce $\mathrm{NO}_{x}$ emissions, and their improper function can lead to increased emissions and power losses [2]. Due to its importance in controlling emissions, on board diagnostic (OBD) requirements have been developed [3] for the EGR that require detection of low flow, high flow, slow response and insufficient cooling malfunctions. Current OBD systems operate on direct sensor measurements and perform a series of limit checks for these known fault scenarios. While simple, this method suffers from several drawbacks,

- Large signal variations, sensor noise and different operating conditions necessitate the use very conservative thresholds [4], possibly resulting in undetected faults. As regulations become more restrictive, this conservativeness becomes a larger issue. 
- Since automotive systems are incorporating more and more interacting dynamic systems (Variable Geometry Turbocharging and EGR [2], for example), a single component fault can result in numerous alarms that can complicate isolation [4].

- Detectable faults are limited to faults that can be enumerated by the system designer. As system complexity increases, this enumeration becomes increasingly cumbersome, perhaps even infeasible.

- No estimate of the fault magnitude is provided, since the OBD output is simply a binary condition. Additionally, if the threshold is not exceeded the system is assumed to be operating normally. Such a hard decision boundary yields no quantification of normality within the (conservative) thresholds.

Clearly, a new methodology that can overcome these shortcomings is desirable. In this thesis, a fault detection and diagnosis (FDD) scheme is designed for a diesel engine EGR system which utilizes a general, data driven framework that can be employed in many types of dynamic systems with little a priori knowledge.

\subsection{Objectives and Challenges}

Automotive fault detection and diagnosis is viewed as a particularly challenging task due to their complex nature, existence of numerous interacting dynamic systems, presence of significant noise levels and the need to deal with numerous and uncertain inputs. The objectives of this thesis are to design an EGR diagnostic system that can: 
- Quantify faults as deviations from normal behavior instead of simply setting thresholds. This will allow incipient faults to be detected before they exceed the more conservative thresholds.

- Detect faults that have never been encountered. Current OBD systems are limited to faults that can be anticipated by the designer. This is not satisfactory since it is impractical to enumerate all the faults that can occur in complex systems, such as an automotive EGR.

- Accomplish precedent-free isolation of systems which caused faulty behavior. The new design should have the capability of isolating the fault to the responsible subsystem without the need to anticipate the fault. The maintenance can then be focused on the faulty subsystem and fault diagnosis and accommodation can be performed more quickly.

- Recognize and characterize faults that have been previously encountered. This will provide an estimate of the faulty system dynamics and a model that can be utilized for control reconfiguration.

The EGR fault detection and diagnosis scheme should also be robust to issues commonly encountered in fault detection, such as noise signal variations as a result of different operating conditions errors. Furthermore, since fault detection and isolation will be conducted using a model-based approach, robustness to modeling errors is necessary to prevent false alarms.

The designed scheme will be tested using a high-fidelity engine simulator, which has detailed models for the air and fuel path. Various faults will be introduced 
to the EGR subsystems and the sensitivity, robustness and simplicity of the FDD scheme will be assessed.

\subsection{Organization of Thesis}

The remainder of the thesis is organized as follows:

- Chapter 2 reviews the state of the art in automotive air path diagnosis.

- Chapter 3 details the fault detection and diagnosis (FDD) methodology based on the recently introduced Growing Multiple Model System (GSMMS) for modeling of complex dynamic systems.

- In Chapter 4 the EGR system is discussed and the corresponding FDD system is designed. Several anomalies are introduced to the EGR subsystems, each is isolated without the need for prior training (precedent-free) and identified using the general GSMMS based methodology.

- Finally, Chapter 5 discusses conclusions and suggests future work. 


\title{
Chapter 2
}

\section{State of the Art for Engine Air Path Diagnostics}

\author{
Automotive diagnostics falls under the larger paradigm of Fault Detection \\ and Diagnosis (FDD). A complete FDD procedure consists of three major subtasks \\ [4]. The first is detection, where a decision is made on whether the system is op- \\ erating normally or not. When a fault is detected, fault isolation is conducted and \\ the faulty subsystem is determined. Finally, fault identification yields a model of \\ the faulty dynamics. Commonly, isolation and identification are together referred \\ to as the diagnosis stage [4]. Usually, identification is not conducted in automotive \\ applications and the procedure is called fault detection and isolation (FDI). In this \\ chapter, methods for FDD for automotive applications are reviewed, with special \\ emphasis on engine air path diagnostics. \\ In automotive literature, FDD methods generally fall into two categories: \\ model-free and model-based [4]. Model-free methods include physical redundancy, \\ limit-checking, or spectrum analysis which operate directly on sensor signals and \\ do not require a mathematical model. This simplicity is the main attraction of \\ model-free methods. The designer anticipates a certain set of faults and sets global \\ limits on the normal operation of the signal. If the monitored signal is within the \\ thresholds, the system is assumed to be operating normally. This approach is the
}


basis of the On-Board-Diagnosis II (OBD II) regulations that require the diagnostic system to check for a pre-described set of faults [3]. However, the setting of model-free thresholds is problematic since normal variation in sensor readings occurs due to noise, changes in the system operating point and other disturbances. This variation requires that model-free decision thresholds are set conservatively [4] to prevent false alarms. This conservatism could result in missed faults, particularly if they occur in operating regions where the normal signal variation is small.

Another issue with the model-free paradigm is the need for extensive a priori knowledge, experience and/or historical data. The system faults must be anticipated to conduct FDD. One must enumerate the possible faults off line and propagate these faults through the interacting dynamic system to the measured signal(s). Clearly for complex systems of numerous interacting dynamic sub-systems (such as automobiles) such enumeration is cumbersome and potentially infeasible. In addition, diagnosis is difficult since many different subsystem faults could result in the monitored signals exceeding their thresholds.

Current EGR OBD methods fall under the model-free paradigm, based on limit checks on raw sensor signals. By running through a series of these checks, the OBD system can detect and isolate a certain set of faulty signals that fit anticipated patterns. However, if the fault signal patterns do not conform to those in the OBD checklist, it is assumed that the system is behaving normally. Due to this requirement for extensive $a$ priori knowledge and aforementioned conservatism, such model-free methods will likely not be able to meet increasingly stringent detection requirements. 
Model-based methods offer a way to enhance detection and facilitate diagnosis by utilizing analytical information to evaluate sensor signals [5]. Many researchers have examined model based methods. Most of the methods applied to automotive fault detection are structured as in Fig. 2.1, with a model characterizing normal behavior and utilizing engine measurement to generate residuals, usually of the form

$$
\mathbf{r}(t)=\mathbf{y}(t)-\hat{\mathbf{y}}(t)
$$

or

$$
\mathbf{r}(t)=\boldsymbol{\theta}_{m}-\hat{\boldsymbol{\theta}}(t)
$$

where $\mathbf{y}(t)$ and $\boldsymbol{\theta}(t)$ are the actual output and current model parameter vector respectively, while $\hat{\mathbf{y}}(t)$ and $\boldsymbol{\theta}_{m}$ are the normal model output and parameter vector respectively. In both cases $|\mathbf{r}(t)| \approx 0$ for normal operation and $|\mathbf{r}(t)|>0$ in the presence of a fault. As with model-free methods, most model-based methods rely

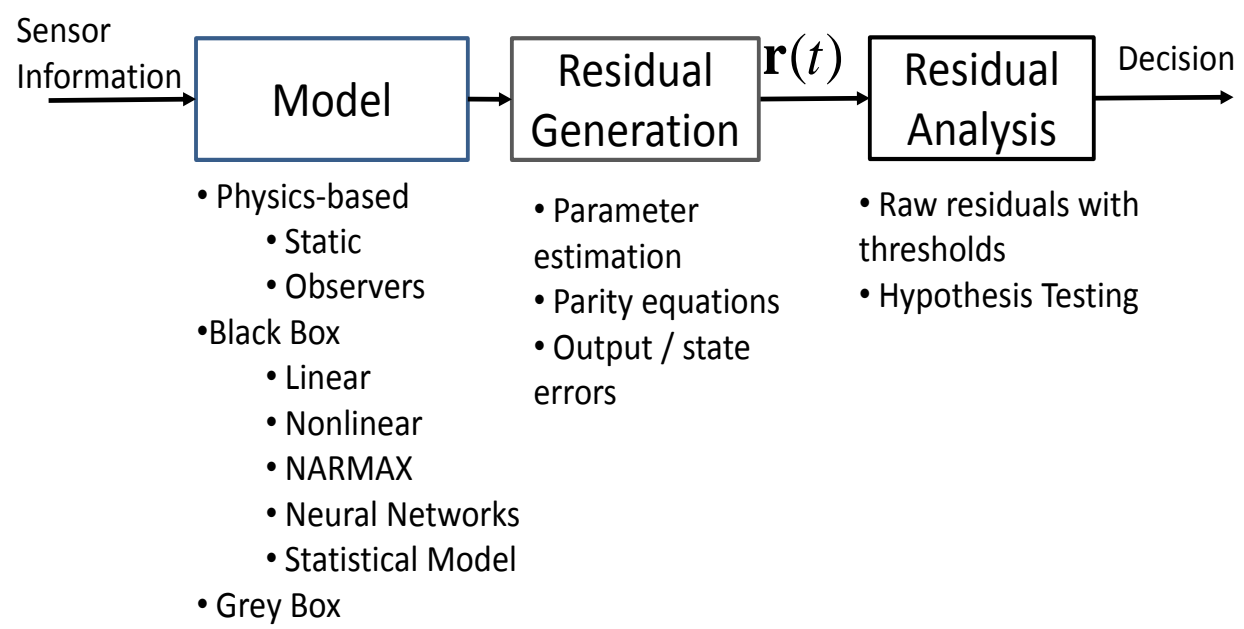

Figure 2.1: Model-based detection in automotive literature. 
on the designer knowledge to connect changes with known faults. Using a priori knowledge, residual signals must be structured to detect a set of faults that the designer deems important. A good design will result in a fault that is detected and isolated based on a fixed set of monitored residuals.

In the following sections, methods for model-based fault detection, isolation and identification in automotive applications will be reviewed.

\subsection{Fault Detection}

Many of the early methods in automotive fault detection utilized linearized dynamic models to detect sensor [6] and process failures [7]. Simplified engine models were utilized and offered suitable accuracy for the desired detection levels.

While the theory of linear fault detection is well developed and theoretically elegant [4], its implementation requires restrictive simplifying assumptions. Foremost is the assumption that the model and its parameters are well known. In practice, parametric errors and unmodeled dynamics affect the FDD scheme by causing surges in modeling errors that lead to false alarms or force one to set overly conservative detection thresholds [8]. To overcome such difficulties, researchers have explored nonlinear models to better account for the numerous nonlinear phenomena present in engines. Conatser et al. used a detailed physical model to detect faults in an electronic throttle system [9]. Mohammadpour et al. [10] utilized a static nonlinear air path model and an online parameter estimation technique to detect and estimate EGR leaks and restrictions. Ding et al. [11] applied a linearized FDI approach to a spark-ignition engine using a nonlinear dynamic process model. 
Cecarelli et al. [12] utilized nonlinear observers (adaptive and fixed-gain) to detect the presence of an air intake leakage by estimating a parameter of a leakage model. Nyberg and Stutte [13] utilized nonlinear air path models in the framework of structured hypothesis tests to classify the current behavior as normal or faulty. Detailed models of normal and faulty behaviors were used and the unknown parameter in the faulty models were estimated using an adaptive observer or Extended Kalman Filter. Kim et al. [14] used a set of sliding mode observers to detect faults in the fuel and air path of an SI engine. All of these methods utilized detailed physical models and exploited the particular mathematical structure of the equations. Thus, they require extensive system knowledge and are highly specialized to the particular detection task.

Other researchers have attempted data driven methods. Antory [15] conducted fault detection using a simplified regression model which utilized the well know methodology of principal component analysis [16] to identify significant regressors. Gertler et al. [7] utilized a heuristically defined discrete polynomial structure with a dynamic first order model and a static quadratic part. Krishnaswami et al. [1] utilized a more general Nonlinear Autoregressive Moving Average with Exogenous inputs (NARMAX) $[17,18]$ to diagnose a suite of engine sensor and input faults. Franchek et al. [19] employed a nonlinear model identified by a process called system probing which used the system frequency response to identify significant regressors. Some researchers $[20,21]$ have successfully applied dynamic neural networks (NNs) to detect air path faults while others $[5,22]$ used physical models to model the dominant phenomena and NNs to model secondary, highly 
nonlinear phenomena.

While the observer, data-driven and statistical model-based methods were successful to some degree, there are some important limitations to these approaches. The physics based approaches give useful insight to the dynamics, but require detailed knowledge of the plant and extensive parameter sets, most of which must be known before hand. The data driven approaches have the potential to yield accurate models, but determining the model structure can be problematic, especially since the underlying structure often lacks physical significance.

\subsection{Fault Isolation}

After a fault has been detected, fault isolation is conducted to identify the faulty subsystem. In automotive FDD, detection and identification are usually done with a single set of residuals, $\mathbf{r}(t)$. During detection, a binary residual "flag" is generated by comparing elements of $\mathbf{r}(t)$ with their respective thresholds, or

$$
f_{i}= \begin{cases}1 & \text { if }\left|r_{i}\right|>\text { threshold } \\ 0 & \text { otherwise }\end{cases}
$$

where $r_{i}$ represents the $i$ th element of $\mathbf{r}$. In $[6,23]$ a set of observers was used to generate a set of residuals similar to Eq. 2.1. Using a set of observers that are only sensitive to a particular subset of the faults to be detected, a unique set of binary flags $\mathbf{f}=\left[f_{1}, f_{2}, \ldots\right]^{T}$ were constructed for isolation of each fault. A similar method is utilized by Gertler et al. [24] and is referred to as parity relations. By creating a set of algebraic consistency relationships (residuals) that are insensitive to particular faults, it was shown that fault isolation could be conducted in the fuel and manifold 
subsystems.

More recently, nonlinear parity equation residual generation (NPERG) [1] has been utilized to isolate a variety of engine faults $[1,5,14,25]$. The NPERG scheme utilizes forward and inverse models to generate a input and output residual signals. If the models and residual signals are constructed intelligently, a suite of input (actuator) and output (sensor) faults can be detected and isolated. The NPERG scheme that Krishnaswami et al.[1] designed is shown in Fig. 2.2 with their isolation logic shown in Table 2.1

\begin{tabular}{c|c|c} 
Fault Location & non-zero $r_{f}$ elements & non-zero $r_{i}$ elements \\
\hline air mass flow sensor & all & all \\
engine speed sensor & engine speed residual & all \\
throttle angle & all & throttle angle residual \\
fuel mass & all & fuel mass residual
\end{tabular}

Table 2.1: Isolation logic utilized in [1]. $r_{f}$ is a vector of residuals of the forward model. Similarly, $r_{i}$ is a vector of residuals of the inverse model.

Another noteworthy approach was that proposed by Nyberg and Stutte [13]. They devised an isolation method using structured hypothesis tests. Using a set of observers, they estimated unknown parameters of the fault models and constructed test quantities from the residuals. These test quantities were then normalized and compared to thresholds to reject a set of hypotheses. It is important to point out that such hypothesis test based isolation required identification of the fault prior to isolation.

All of the aforementioned methods are inference methods [26] where the designer maps the symptoms (residual signals) to faults. Some researchers have 


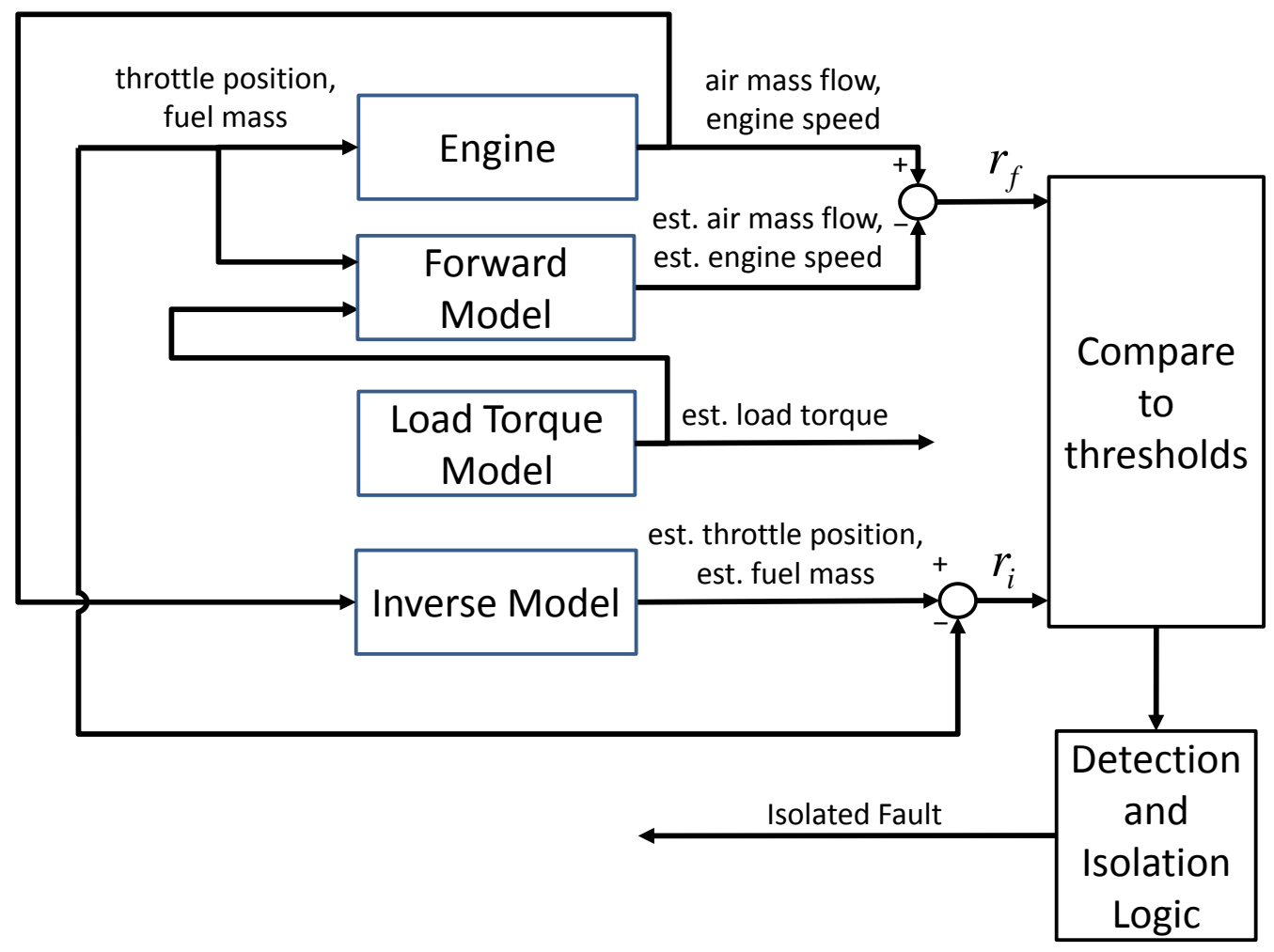

Figure 2.2: Engine NPERG scheme utilized in [1]. The forward and inverse NARMAX models are utilized to generate residual vectors $r_{f}$ and $r_{i}$ which are used in the fault isolation logic (see Table 2.1.) 
applied fuzzy inference [25] to utilize heuristic and analytical system knowledge for isolation. Another class of isolation systems are called classification methods. An application of fuzzy classification can be found in [27] where a vehicle level fuzzy classifier used information from signal diagnostic agents to isolate the faulty subsystem. Such classification methods have rarely been applied in automotive diagnosis. This is likely due to the fact that they require extensive faulty data sets to train the classifier to diagnose each faulty condition.

On the other hand, geometric classifiers have been applied with some success [19], but still require one to define "fault assessment vectors" in the residual space. These vectors "point" in the direction of a normalized $\mathbf{r}(t)$ that is indicative for a given fault. One then projects the residuals $\mathbf{r}(t)$ onto each of the fault vectors, and the projection with the largest magnitude is selected as the fault. While simple and easily implemented, the system knowledge required is similar to that of parity relations. One must know the elements of $\mathbf{r}(t)$ that will be nonzero for each fault.

From the discussion above, it can be seen that the state of the art in automotive fault isolation involves

- Selecting a subset of faults that are to be detected.

- Design of a classifier or a set of residual equations that allow isolation of the above faults.

- Recording of residuals and their comparison to thresholds to account for system noise, modeling errors, external disturbances. 
- Use of a designed logic set (or classifier) to determine the fault location.

\subsection{Fault Identification}

Fault identification is the second part of the diagnosis function (fault isolation and identification are the elements of diagnosis), whose goal is to estimate a model for the faulty system. In automotive FDD, this "identification" has been taken as the more modest objective of estimating the "magnitude" of the fault [4]. In this sense, magnitude refers to the degree of deviation from normal behavior with smaller magnitudes being closer to normal behavior. In automotive applications, this estimation is usually conducted in one of two ways: utilizing output residuals or utilizing fault models. Outside of these methods, researchers have rarely conducted fault identification [19], usually being satisfied with determining the location of the faulty component or subsystem.

The subject of fault identification received little attention in automotive literature. There are a few likely reasons for this. First, model-based detection has proved difficult for automotive applications due to the inherently complex and nonlinear nature of the underlying systems. This has made the modeling task difficult enough that finding a model (or models) suitable for detection drew much of the research attention [28]. Second, in the early 1990's manufacturers were focused on meeting upcoming OBD II regulations which required detection of sensor failures and a subset of process failures. This deadline likely focused research on utilizing methods that would enable manufacturers to meet these regulations $[6,7]$. Since

there were no requirements for fault identification in OBD II, it was not a major 
research focus. Finally, since the on board computing power was very limited [28], researchers have focused on keeping the computational burden low . Adding fault identification would increase the computational burden and the benefits of fault estimation were not worth the increased cost associated with the increase in computational complexity.

However, as on-board computational capacity has increased [29], more researchers have included some type of fault identification in their proposed FDD schemes. One type of fault identification scheme that has been employed is the use of detailed fault models. Nyberg and Stutte [13] utilized detailed fault models with parameter estimation in the framework of structured hypothesis tests. In this situation, the hypothesis test returns a statement that instantaneously yields detection, isolation and identification in one, essentially conducting all three FDD tasks in parallel. However, this parallelism is at the cost of extensive modeling effort and detection of only a limited set of faults.

Another fault identification method is to utilize the residual signal and assume that the fault is the manifestation of additional multiplicative uncertainty as suggested in [19]. More precisely, the form

$$
y_{\text {fault }}(t)=(1+\Delta) y_{\text {model }}(t)
$$

is used, where $\Delta$ is the fault effect and was calculated as

$$
\Delta=\frac{y_{\text {fault }}(t)-y_{\text {model }}(t)}{y_{\text {model }}(t)}=\frac{r(t)}{y_{\text {model }}(t)}
$$

This scheme was effective primarily because fault detection was only conducted when the engine was near steady-state and thus one could model the faults as 
changes in steady-state gain. A similar method was employed to estimate the fault size in [10], where Eq. 2.4 was used to estimate the size of an EGR leak or restriction. 


\section{Chapter 3}

\section{GSMMS Based Anomaly Detection, Isolation and Identification}

This chapter will discuss an integrated framework for the detection, isolation and identification of faults developed by Liu [30]. The foundation of this framework is the Growing Structure Multiple Model System. The GSMMS method for modeling of dynamic systems provides a generic, flexible and easily interpretable model structure that enables modeling of a wide variety of dynamic systems. Furthermore, the piecewise model structure of the GSMMS naturally leads to the operating-point dependent interpretation of residuals for the purpose of more reliable anomaly detection.

\subsection{The Growing Structure Multiple Model System (GSMMS)}

An important decision in FDD is the choice of the model for the characterization of the behavior of the system. The accuracy of the modeling approach will determine the sensitivity of the fault detection, since more accurate models can have tighter decision thresholds [1]. Many researchers have utilized detailed physicsbased models (see $[9,10,23,31]$, for example), but such models require extensive $a$ priori knowledge and their simplicity often limits their detection of more complex 
faults. For this reason, other researchers have pursued semi-physical [5] or datadriven approaches $[1,19,20]$ with varying degrees of accuracy and computational burden. The GSMMS approach discussed in this section is a dynamic, data-driven modeling approach and will underlie the EGR FDD strategy presented later.

\subsubsection{The GSMMS Modeling Approach}

Various data driven modeling methods have been used for identification of complex dynamic systems. They impose fewer assumptions on the underlying model while requiring less a priori knowledge compared to the first princi-

ple physics based models. Methods such as Multi-Layer Perceptrons (MLPs) and Radial Basis Function (RBFs) have been used extensively for data driven modeling due to their universal approximation capabilities [32]. However, determining the structure (number of layers, number of hidden neurons) remains a challenging problemand the commonly used training algorithms often require extremely lengthy training times [32].

Both MLPs and RBFs utilize training data from the entire operating space and can thus be considered "global" models [33]. An alternative to such global modeling methods is the "divide and conquer" approach where the operating space is decomposed into smaller sub-regions. A variety of frameworks have been proposed for such multiple model systems. Takagi and Sugeno [34] proposed a model that creates a fuzzy partition of the input space using membership functions. Although the Takagi-Sugeno Fuzzy Model (TSFM) can apply well known methods (such as the Kalman Filter [35]) to estimate the local model parameters, the model 
structure (premise variables) must be determined using a heuristic. The problem of determining both local parameters and model structure is addressed by NeuroFAST [36] which utilizes fuzzy adaptive resonance theory along with fuzzy rule splitting and addition to provide better coverage of regions that are difficult to model. An approach proposed by Johansen and Foss [37] computes the global output using the following structure:

$$
\begin{array}{r}
y=\sum_{i=1}^{M} v_{i}(x) f_{i}(x) \\
v_{i}(x)=\frac{\rho_{i}(x)}{\sum_{i=1}^{M} \rho_{i}(x)}
\end{array}
$$

where $f_{i}(x)$ is the local model, $y$ is the output and $\rho_{i}(x)$ describes the validity of the $i^{\text {th }}$ model. The global output can be seen as a weighted sum of the local models. However, the regime decomposition relies on heuristics and the centers for the validity functions are limited to a rectangular grid. For this model and the TSFM, the problem of determining the structure is computationally complex.

Instead of dividing the individual input variables, vector quantization techniques, such as a Kohonen's Self-Organizing Map (SOM) [38], have been proposed to identify a dynamic model structure [33] [39]. The set of weight vectors $\boldsymbol{\xi}_{i}, i=1, \ldots, M$ of a SOM define a Voronoi Tessellation:

$$
V_{m}=\left\{x:\left\|x-\boldsymbol{\xi}_{m}\right\| \leq\left\|x-\boldsymbol{\xi}_{j}\right\|, \forall j \neq m\right\}
$$

Equation 3.2 defines a partition of the state space by using the structural parameters $\xi_{i}$ to create sets of $x$-s that are closest to each vector in the Euclidian sense. A two dimensional version of the Voronoi Tessellation can be seen in Fig. 3.1. 


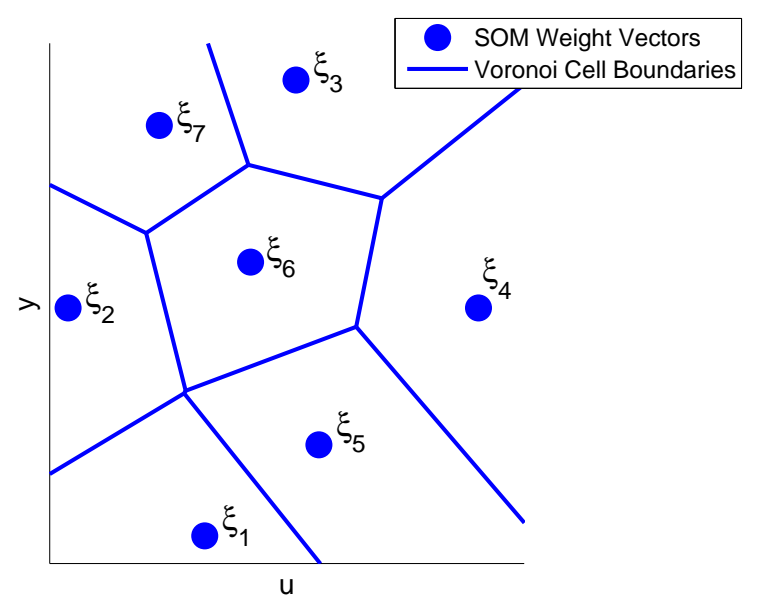

Figure 3.1: Voronoi Tessellation using 7 weight vectors $\xi_{i}$.

If a SOM is used to cluster vectors consisting of inputs and outputs of a dynamic system, the Voronoi Tessellation ends up partitioning the input-output space into sub-regions of "similar" input-output patterns [40]. An advantage of this method is that the partition is adjusted through unsupervised clustering carried out by modifications of the SOM. In this respect, the SOM acts as a set of "decoders" to order the space [38]. However, the number of nodes and the number of topological connections still need to be determined in advance.

Recent developments in growing SOMs allow the maps to grow to appropriate size and impose fewer assumptions on the underlying data. Growing neural gas [41], growing cell structures [42] and growing SOMs [43] incorporate specific addition and deletion mechanisms to allow the SOM to determine their appropriate order and quantity.

These developments led to the recently introduced GSMMS [30] approach 
to dynamic system modeling. It utilizes a growing SOM to decompose the inputoutput space into sub-regions with similar dynamic behavior and to refine the partition in poorly modeled regions. More specifically, the GSMMS uses the multiple models structure of Eq. 3.1, where each of the $M$ regions are defined by the SOM induced Voronoi Tessellation. Local models are assumed to be of the affine form

$$
\mathbf{F}_{m}(\mathbf{s}(k))=\mathbf{a}_{m}^{T} \mathbf{s}(k)+\mathbf{b}_{m}
$$

with $\mathbf{a}_{m}$ and $\mathbf{b}_{m}$ denoting the parameters of the local model $m$ and

$$
\begin{array}{r}
\mathbf{s}(k)=\left[\mathbf{y}^{T}(k), \ldots, \mathbf{y}^{T}\left(k-n_{a}+1\right), \mathbf{u}^{T}\left(k-n_{d}\right), \ldots,\right. \\
\left.\mathbf{u}^{T}\left(k-n_{d}-n_{b}+1\right)\right]^{T}
\end{array}
$$

where $\mathbf{y}(k)=\left[y_{1}(k), \ldots, y_{n}(k)\right]^{T}$ and $\mathbf{u}(k)=\left[u_{1}(k), \ldots, u_{p}(k)\right]^{T}$ for a system with $n$ outputs and $p$ inputs, $n_{a}$ and $n_{b}$ are the autoregressive and input orders respectively and $n_{d}$ is the delay. As in Johansen and Foss [37], the global model is then defined as an interpolation of local models

$$
\hat{y}(k+1)=\sum_{m=1}^{M} v_{m}(\mathbf{s}(k)) \mathbf{F}_{m}(\mathbf{s}(k))
$$

where $v_{m}(\mathbf{s}(k))$ is the function describing the way local models are mixed into the global model. Following Liu [44], $v(\mathbf{s}(k))$ is taken to be a simple gating function

$$
v_{m}(\mathbf{s}(k))=\left\{\begin{array}{cl}
1 & \mathbf{s}(k) \in V_{m} \\
0 & \text { otherwise }
\end{array}\right.
$$

which defines the region of validity of each local model as one of the Voronoi sets of the underlying SOM. Subsequently, this structure implies that only one local model is used to describe the dynamics at a given sampling instant, $k$. 
One can see that the GSMMS essentially casts the problem of representing the system dynamics into the framework of simple, interconnected dynamic linear models. This structure enables the modeling of a wide variety of complex systems while maintaining analytical tractability. Local model tractability recently led to important results in terms of global characteristics of the model parameter estimation during learning [45], model stability [46] and is expected to lead to describable control capabilities [47]. The GSMMS has been used successfully for modeling of an electronic throttle system in a gasoline engine [44] and automotive crankshaft dynamics [48].

\subsubsection{Training of the GSMMS}

To train the GSMMS using data emitted from the system of interest, Liu et al. [44] utilized a sequential algorithm suitable for online training and briefly discussed modifications for so-called "batch" training when all the training data is available. For this thesis, all of the training data was available at once, so batch training could be conducted.

In order to model a system using the GSMMS, both the structural parameters of the model (weight vectors) and local model parameters must be determined. The input-output space partition is updated by adjusting the SOM weight vectors,

$\boldsymbol{\xi}_{m}, m \in\{1,2, \ldots, M\}$. The SOM weight vectors are updated using the the recursive updating relation

$$
\boldsymbol{\xi}_{m}(k+1)=\boldsymbol{\xi}_{m}(k)+\zeta_{m}(k) \sum_{b=1}^{M} h(k, \operatorname{dis}(m, b))\left[\overline{\mathbf{s}}_{b}-\boldsymbol{\xi}_{m}(k)\right]
$$




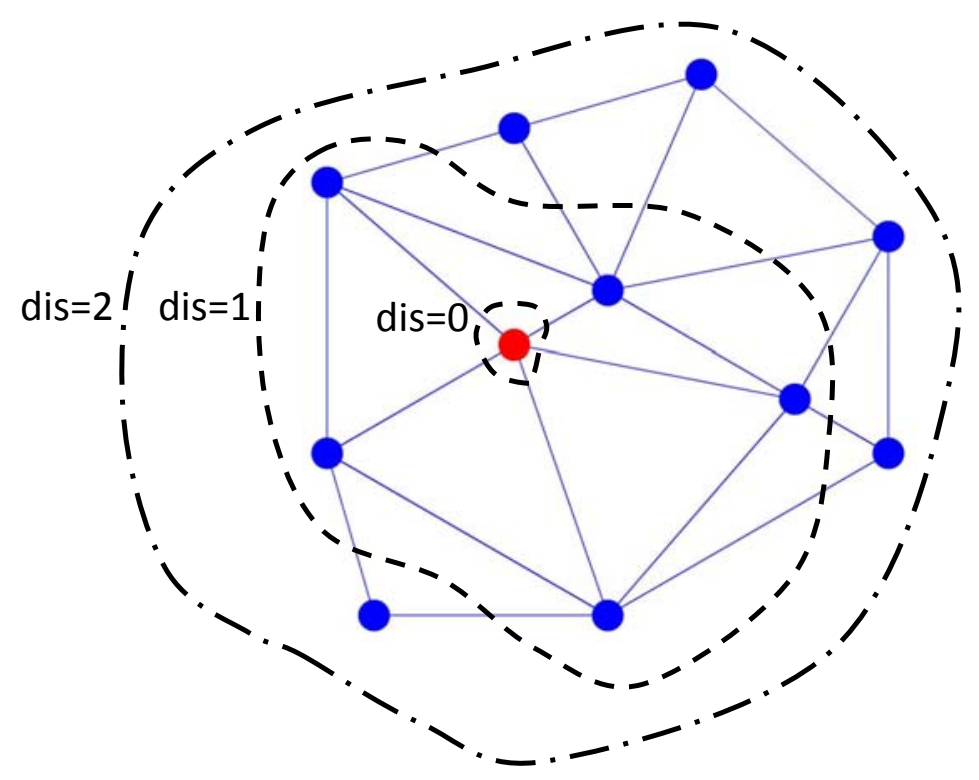

Figure 3.2: Example of the topological distance calculation, $\operatorname{dis}(m, b)$. The the dashed contours enclose nodes that are the respective distances away from the Best Matching Unit (dis=0). Each of the nodes are the center of a model (Voronoi region).

where $k$ is the epoch number (number of passes through the training data), $\operatorname{dis}(m, b)$ is the topological distance between regions $m$ and $b$ computed using the Breadthfirst procedure [49] as depicted in Fig. 3.2 and $\overline{\mathbf{s}}_{b}$ is the sample mean of the training vectors for which $b$ is the Best Matching Unit (BMU). For each training item, $\mathbf{s}(i)$, the (BMU) is index of the local model for which $\mathbf{s}(i) \in V_{m}$, or

$$
b(i)=\arg \min _{m}\left\|\mathbf{s}(i)-\boldsymbol{\xi}_{m}\right\|
$$

The function $h(k, \operatorname{dis}(m, b))$ is the neighborhood function that enables each vector to be updated using training samples in neighboring regions. A typical form for this 
neighborhood function is

$$
h(k, \operatorname{dis}(m, b))=\exp \left(\frac{-\operatorname{dis}(m, b)^{2}}{2 \sigma^{2}(k)}\right)
$$

where the width parameter $\sigma(k)$ is usually taken to be larger in the initial training stages and tends to zero as $k \rightarrow \infty$ to achieve convergence and global ordering of the SOM [50]. In this thesis, $\sigma(k)$ was taken to be

$$
\sigma(k)=1 / k
$$

Since the ultimate goal of the SOM in the GSMMS is to yield accurate modeling, the updating of SOM nodes described by Eq. 3.7 is augmented with a penalty term

$$
\zeta_{m}(k)=\frac{e_{m}(k)}{\sum_{m=1}^{M} e_{m}(k)}
$$

where $e_{m}(k)$ is

$$
e_{m}(k)=\sqrt{\frac{1}{k} \sum_{i=1}^{k}[y(i)-\hat{y}(i)]^{2}}
$$

or the root mean square (RMS) modeling errors in the $m$ th region. By examining Eq. 3.7, one can see that due to the term $\zeta_{m}(k)$, the weight vectors will tend to move toward regions with higher modeling errors. Since the SOM nodes are centers of the local models this movement ultimately leads to a finer partition of the input-output space in areas of high nonlinearity.

The local model parameters in region $m$ are determined by minimizing

$$
\min _{\boldsymbol{\theta}} J_{m}\left(\boldsymbol{\theta}_{m}\right)=\frac{1}{2 K}(\mathbf{Y}-\mathbf{S} \boldsymbol{\theta})^{T} \mathbf{W}_{m}(\mathbf{Y}-\mathbf{S} \boldsymbol{\theta})
$$


where

$$
\begin{gathered}
\boldsymbol{\theta}=\left[\begin{array}{l}
\mathbf{a}_{m} \\
\mathbf{b}_{m}
\end{array}\right] \in \mathbb{R}^{r+n \times n} \\
\mathbf{Y}=\left[\begin{array}{cccc}
y(1) & y(2) & \ldots & y(K)
\end{array}\right]^{T} \in \mathbb{R}^{K \times n} \\
\mathbf{S}=\left[\begin{array}{cccc}
\mathbf{s}(1) & \mathbf{s}(2) & \ldots & \mathbf{s}(K) \\
1 & 1 & \ldots & 1
\end{array}\right]^{T} \in \mathbb{R}^{K \times r+1} \\
\mathbf{W}_{m}=\left[\begin{array}{llll}
w_{m}(\mathbf{s}(1)) & w_{m}(\mathbf{s}(2)) & \ldots & w_{m}(\mathbf{S}(K))
\end{array}\right] \mathbf{I}_{r+1 \times K}
\end{gathered}
$$

which is a vector formulation of the typical residual sum of squares criterion. Here, $K$ is the number of training samples, $r$ is the dimension of $s(k), n$ is the number of outputs and the ones in $\mathbf{S}$ are to account for the bias term, $\mathbf{b}_{m}$. The function $w_{m}(\mathbf{s}(i))$ determines the weight of the modeling error associated with sample $i$ for the cost function in region $m$, allowing each training sample $\mathbf{s}(i)$ to update a neighborhood of regional models. The weighting function is defined as

$$
w_{m}(\mathbf{s}(i))=\exp \left(\frac{-\operatorname{dis}(m, b(k))^{2}}{2 \sigma(k)^{2}}\right)
$$

so that training samples have a larger effect on the model parameters near the BMU and a smaller effect farther away from the BMU. This cooperation allows the GSMMS to utilize each training sample to affect all local models. Such regional cooperation has been shown to have the ability to speed up the convergence in the early stages of training, but also increase the parameter estimation bias in later training stages [45] (which is why $\sigma(k)$ was reduced to narrow $w_{m}$ with each subsequent epoch). For batch training, the solution to the minimization problem described in Eq. 3.11 is the weighted least squares solution [51]

$$
\boldsymbol{\theta}_{m}=\left(\mathbf{S}^{T} \mathbf{W}_{m} \mathbf{S}\right)^{-1} \mathbf{S}^{T} \mathbf{W}_{m} \mathbf{Y}
$$


After a predetermined number of passes through the training data, $\mathrm{n}_{\text {add }}$, the local model with the highest modeling error is selected. The input-output space partition is then refined by adding a node near this poorly modeled region. As illustrated in Fig. 3.3, the new node is inserted in between the region with the largest modeling errors and its furthest immediate neighbor (in the Euclidian sense). The growth mechanism essentially follows the growing cell structure method proposed in [42] and [43], but uses modeling errors instead of visitation frequency or quantization errors as an insertion criteria.
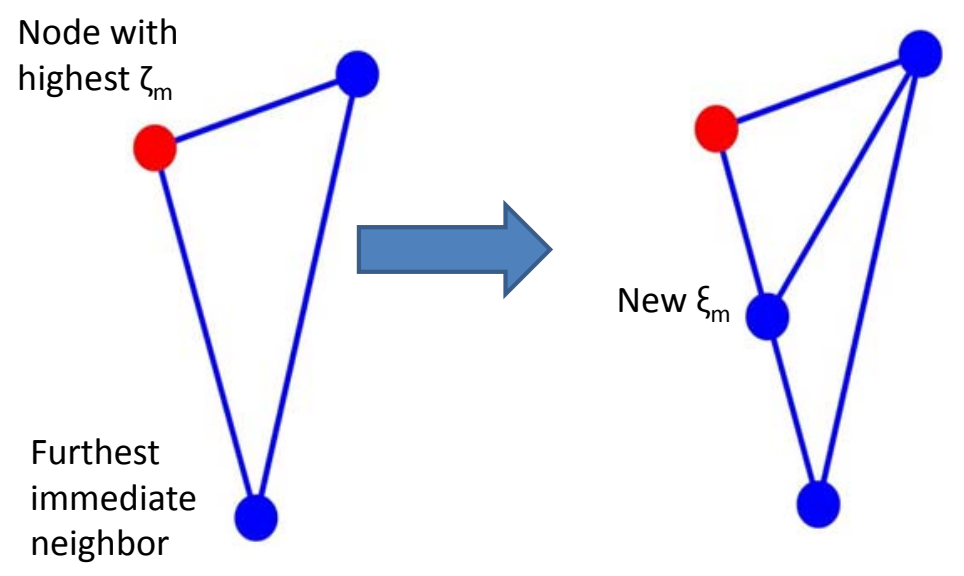

Figure 3.3: Demonstration of growing of the SOM in the GSMMS.

Finally, a stopping criteria is applied to terminate the training. In this paper, two stopping criteria were used: If the total RMS error is below a pre-determined tolerance, or if the number of SOM nodes exceeds a pre-determined number. A flowchart for GSMMS training can be seen in Fig. 3.4. 


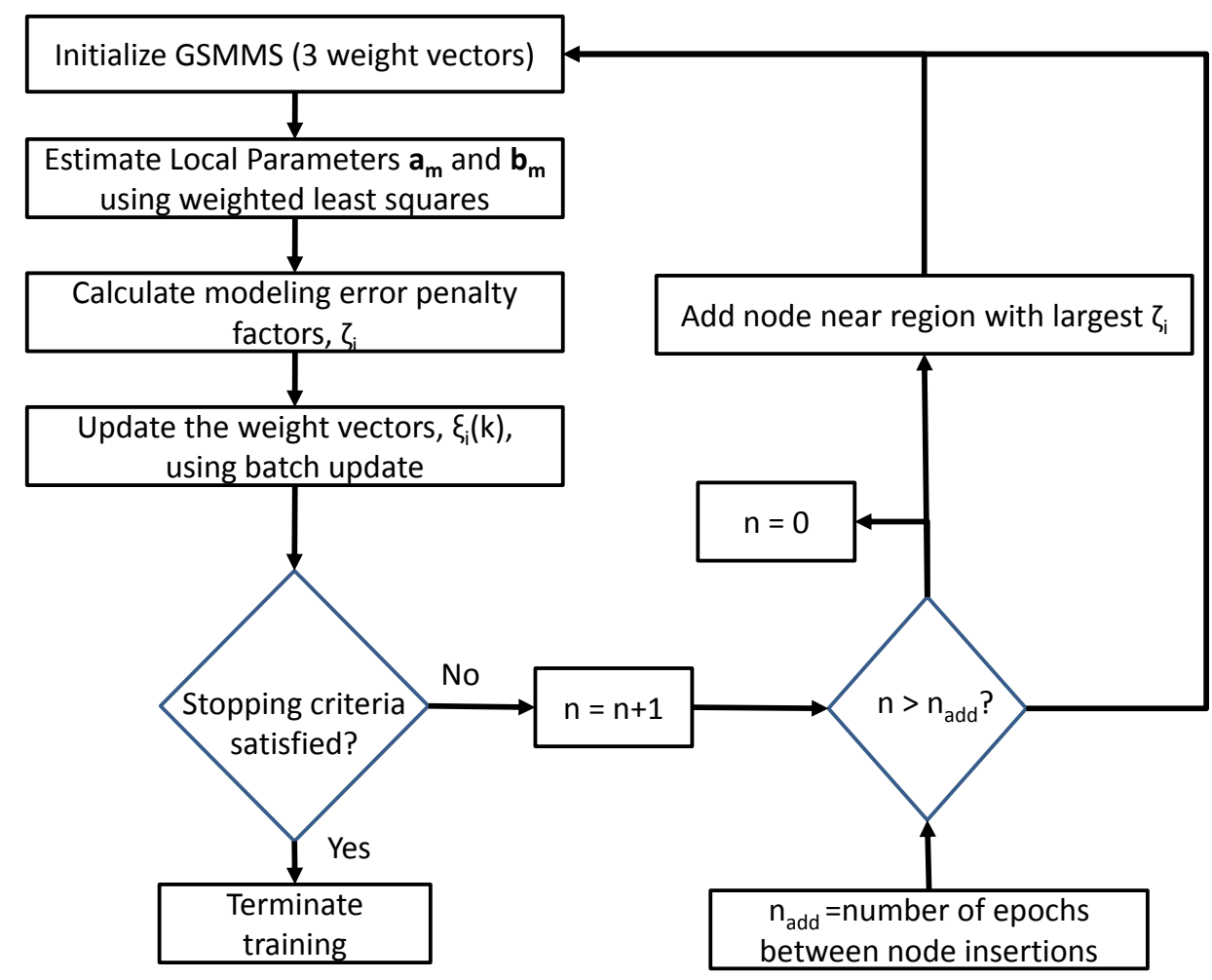

Figure 3.4: Flowchart for GSMMS training.

\subsection{Analysis of Residuals for Fault Detection}

Anomaly detection is accomplished through comparison of the statistical characteristics of the GSMMS modeling residuals displayed during normal behavior with the characteristics of the most recent residuals. The modeling residuals are defined as differences between the system output and the output of the GSMMS describing the normal system behavior. Clearly, one would expect changes in the system dynamics to result in changes in the behavior of the modeling residuals. However, interpretation of the residuals is not a trivial task. A GSMMS model, like 
any other "divide and conquer" model, has regions with different levels of approximation accuracy. Thus, surges in the modeling residuals could occur due to changes in the operating region alone, resulting in false alarms. Therefore, it is necessary to have an anomaly detection strategy that accounts for the regionally dependent residual structures.

An important advantage of the multiple model system framework is that the state space is decomposed into regions of similar dynamic behavior. Just as the GSMMS decomposes the modeling into simpler local models, a simpler residual interpretation can be conducted regionally. Thus, each region of the GSMMS is equipped with its own decision making scheme that quantifies how close the current residual pattern is to the normal pattern.

Following [47], the performance within each region $m$ was quantitatively described using the concept of regional confidence values (CVs) defined as

$$
\mathrm{CV}(m, k)=\frac{\left\langle f_{m}(e) \cdot g_{m}(e, k)\right\rangle}{\left\|f_{m}(e)\right\|\left\|g_{m}(e, k)\right\|}
$$

where $f_{m}(e)$ is the probability density function (PDF) of the modeling residuals displayed during normal behavior and $g_{m}(e, k)$ is the PDF of the residuals corresponding to the current behavior at time $k .|\cdot|$ denotes the inner product

$$
\left\langle f_{m}(e) \cdot g_{m}(e, k)\right\rangle=\int_{-\infty}^{\infty} f_{m}(e) g_{m}(e, k) d e
$$

and $\|\cdot\|$ is the $L_{2}$ norm,

$$
\left\|f_{m}(e)\right\|=\sqrt{\int_{-\infty}^{\infty}\left[f_{m}(e)\right]^{2} d e}
$$




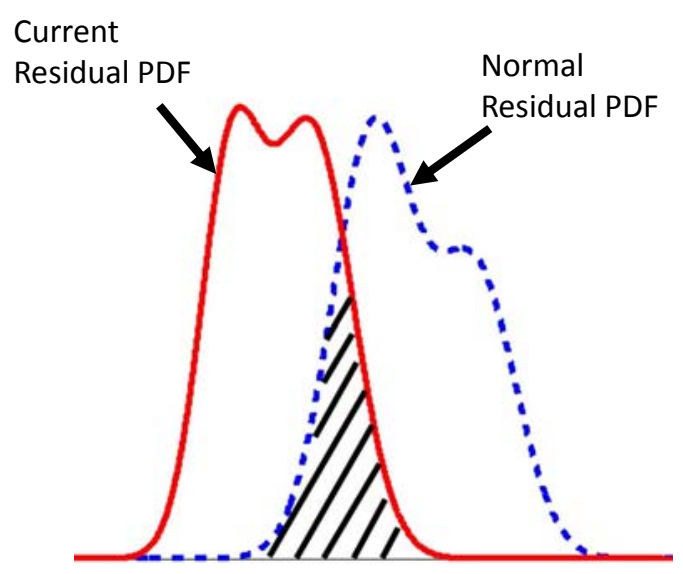

Figure 3.5: Illustration of the interpretation of the Confidence Value.

The regional confidence value, $\mathrm{CV}(m, k)$, describes a normalized area of overlap of the PDFs in that region. This concept is illustrated in Fig. 3.5. It is easy to see from Eq. 3.14 that $\mathrm{CV}(m, k)=1$ if the current residual PDF is exactly the corresponding normal behavior PDF and less than 1 otherwise. The PDF $f_{m}(e)$ was approximated using Gaussian Mixture Models due to their universal approximation capability [52] and $g_{m}(e, k)$ was calculated by updating $f_{m}(e)$ recursively during operation [53]. It should be noted that when a Gaussian Mixture Model formulation is used, a closed form solution for (3.14) can be obtained to quickly compute $\mathrm{CV}(m, k)$ at each time step $k$.

To simplify the monitoring scheme, the regional CVs were merged into a global CV defined as the geometric mean

$$
\mathrm{CV}_{\text {global }}(k)=\left(\prod_{m=1}^{M} \mathrm{CV}(m, k)\right)^{1 / M}
$$

. This was done to emphasize individual departures from normal behavior $(\mathrm{CV}=1)$ 
since a decrease in any $\mathrm{CV}$ indicates that the system is behaving abnormally (at least, in the region where the local CVs are low).

\subsection{Anomaly Isolation Through Distributed Anomaly Detection}

Isolation of the anomaly source can be conducted by reconfiguring and reconnecting anomaly detectors (ADs) to subsystems in the anomalous system. This approach is depicted in Fig. 3.6 for a generic system. The overall AD of the anomalous system is replaced with ADs that monitor the constituent subsystems. Only the CVs output by the AD monitoring the anomalous subsystem will be low. Clearly, for complex systems of interacting subsystems, this process could be repeated until anomalous subsystems are isolated to the smallest possible granularity as illustrated in Fig. 3.6.

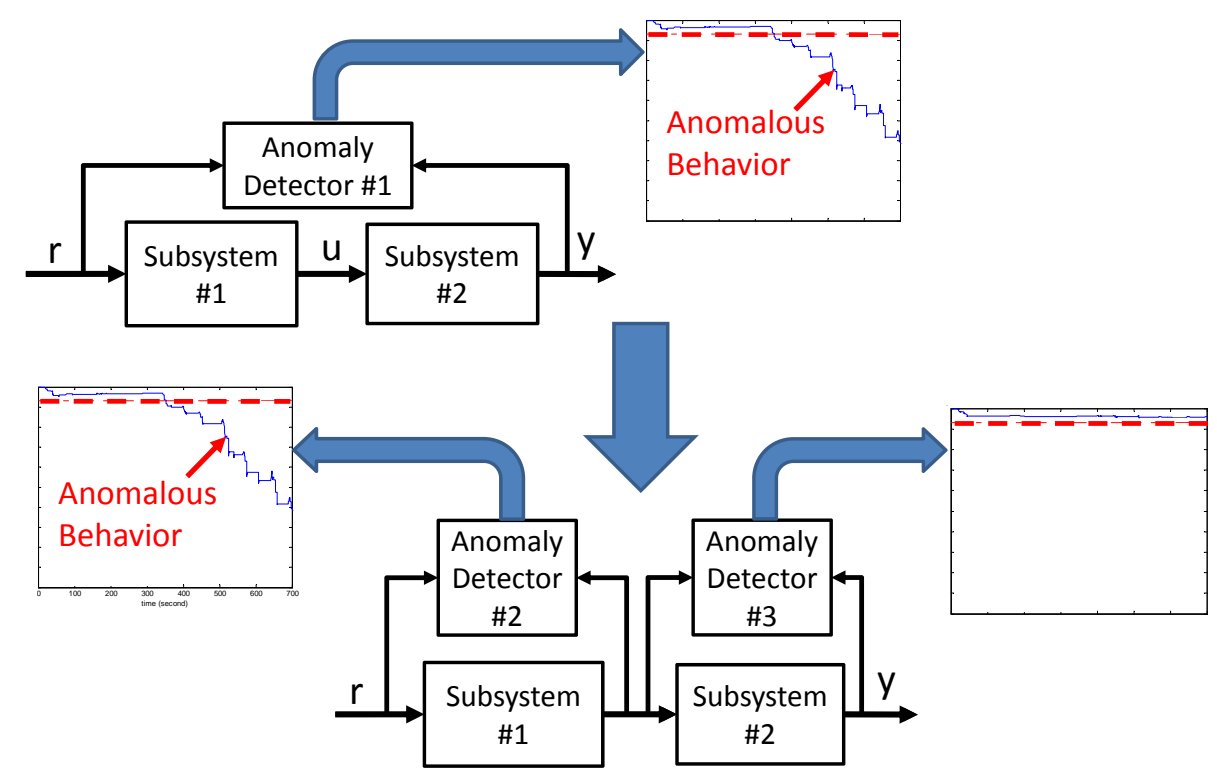

Figure 3.6: Illustration of fault isolation through distributed anomaly detection. 
It should be emphasized that the above isolation logic does not require the "symptom-cause" connections as other schemes utilized in previous engine FDD (see chapter 2). Furthermore, no faulty data is required to train the isolation scheme. Only normal behavior models for the constituent subsystems are required to decide which systems are behaving abnormally. Thus, the anomaly detection is repeated on increasingly basic subsystems until the greatest possible (or desired) granularity is achieved.

\subsection{GSMMS Based Fault Identification}

Once the anomaly is isolated, the next step is to determine whether the detected anomaly has been observed before based on a set of corresponding training signatures, or if the fault is an unknown fault that has never been encountered. As stated in Section 3.2, anomaly detection is accomplished by comparing current system behavior to a GSMMS of normal behavior and computing CVs defined by Eq. 3.14. The same approach can be applied to construct a diagnoser for a specific fault. Data emitted in the presence of a certain fault can be used to train a GSMMS model and the region dependent modeling residual PDFs in the presence of the fault can be estimated. Presence of that fault can then be detected when the most recent modeling residuals generated by the corresponding GSMMS match the modeling residuals observed during training of that GSMMS. Following [44], the similarity of modeling residuals can be evaluated in terms of the appropriate diagnoser $\mathrm{CV}$.

As depicted in Fig. 3.7, this process is similar to how the CVs corresponding to normal behavior are evaluated based on the overlaps between the most 
recent modeling residuals and those generated by the normal behavior GSMMS. Essentially, in the presence of a previously known and modeled fault, the appropri-

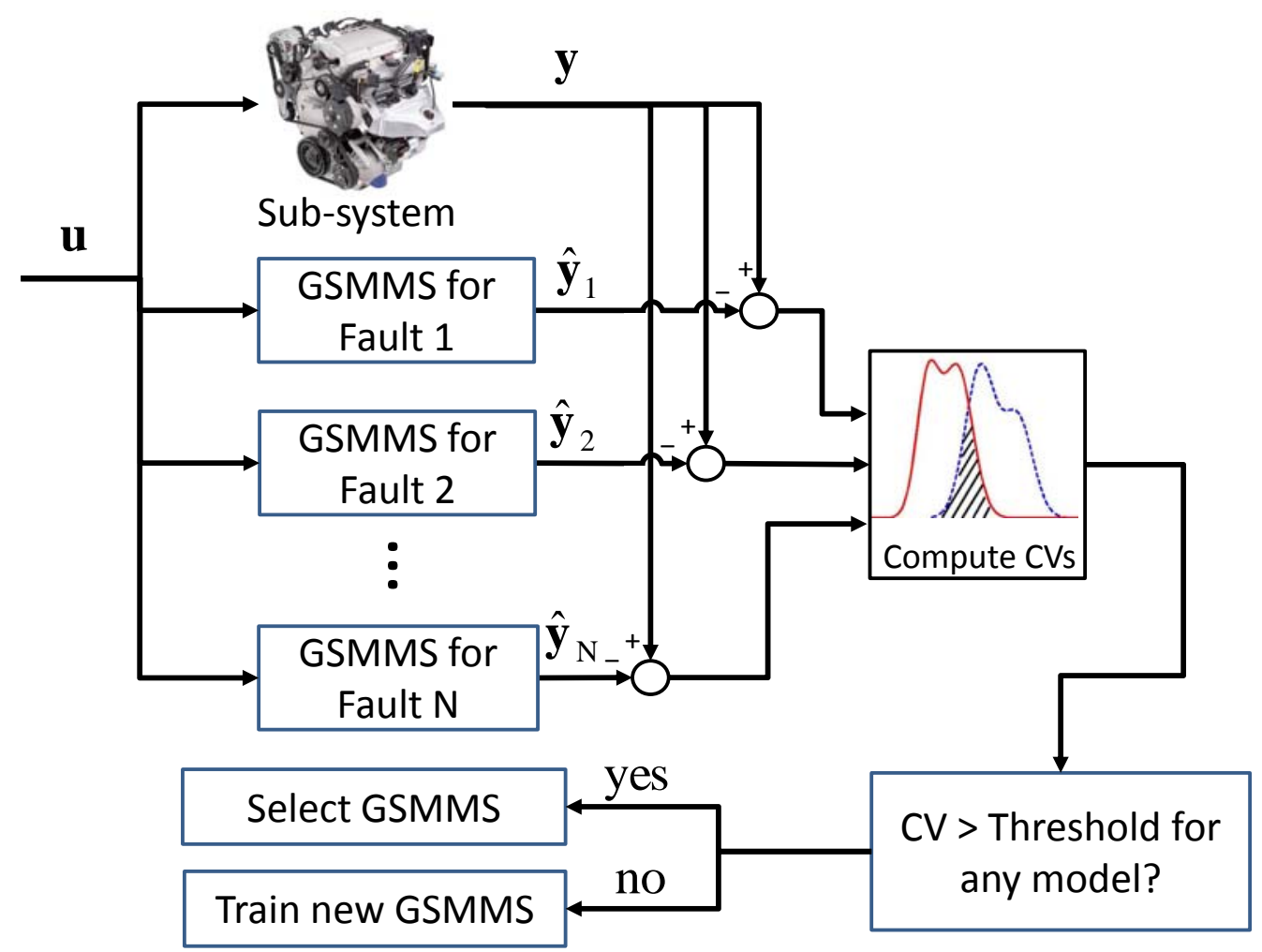

Figure 3.7: Depiction of the GSMMS based fault identification strategy. In the above example, $\mathrm{N}$ faults can be identified.

ate diagnoser will display high CV levels (its modeling residuals match well with those observed in the presence of that fault), while all others will display low CVs. If none of the diagnosers are able to identify its corresponding fault with a high degree of confidence (all CVs are low), the fault is unknown and can be diagnosed in the future by training a new GSMMS for the currently observed system behavior. While it is clear that CV thresholds must still be set, the CVs provide a continu- 
ous assessment of how well a given model matches the current behavior, enabling decisions beyond a "fault / no fault" statement used in previous automotive FDI methods. 


\section{Chapter 4}

\section{A GSMMS Based Approach to Exhaust Gas Recirculation Diagnostics}

Exhaust Gas Recirculation (EGR) is widely used as a method to reduce $\mathrm{NO}_{\mathrm{x}}$ emissions. In an EGR system, a portion of the exhaust gas is introduced into the intake. This proportion is controlled by the EGR valve, which is set by the engine control unit based on the current operating conditions (engine speed and load). A simplified schematic of the EGR system is shown in Fig. 4.1 and the block diagram of the EGR system is shown in Fig. 4.2. In this chapter, a GSMMS based diagnostic

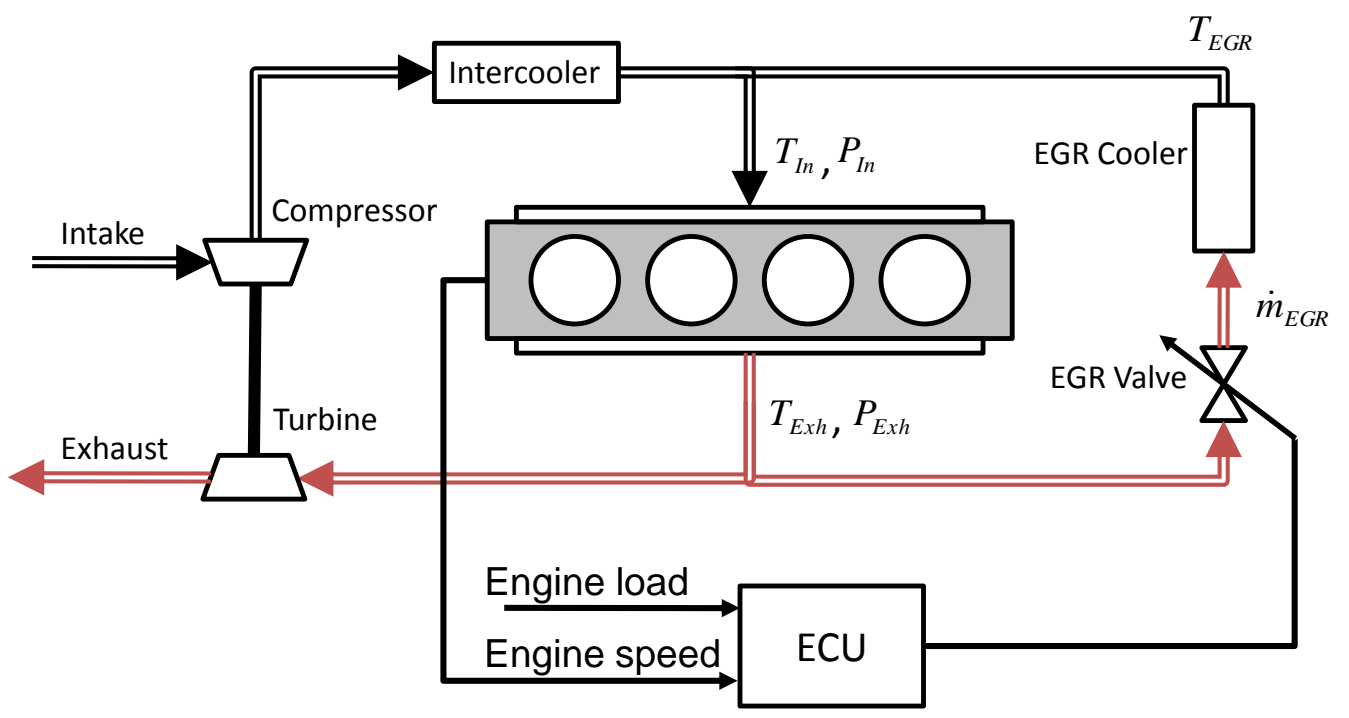

Figure 4.1: Schematic of a generic EGR system. 


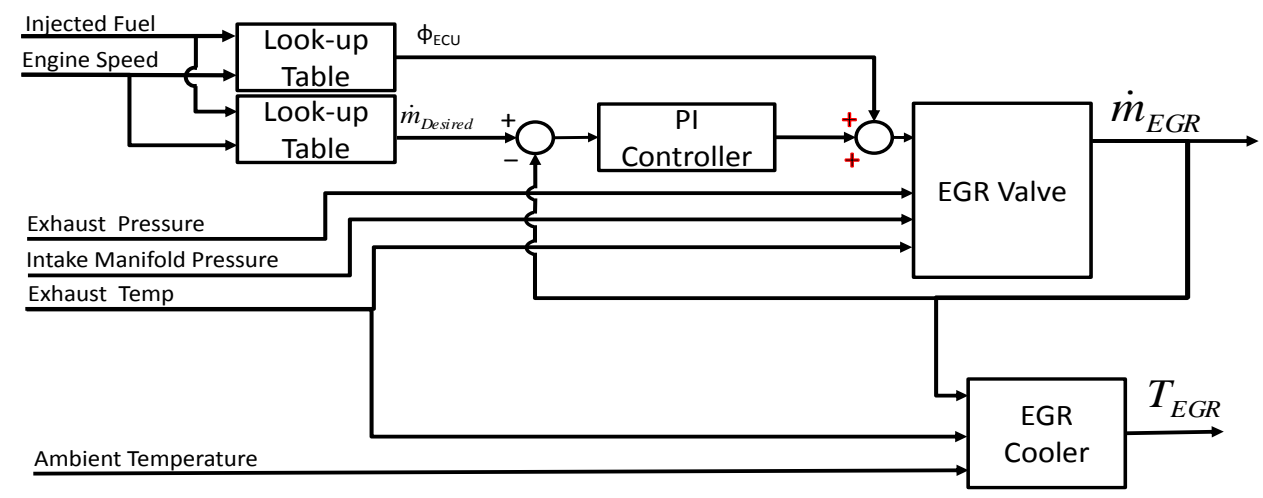

Figure 4.2: Block diagram of the EGR valve system.

approach is applied to a diesel engine EGR system. This approach is able to detect and isolate faults in the controller, cooler and valve subsystems without the need for models of faults or data emitted by faulty systems. Additionally, previously encountered faults are diagnosed using the GSMMS based approach described in Section 3.4.

\subsection{Simulation Model and Fault Descriptions}

The diesel engine dynamics were simulated using the software package enDYNA $^{\circledR}$ THEMOS $^{\circledR}$ CRTD 2.0 by TESIS ${ }^{1}$. En-DYNA is a Simulink ${ }^{\circledR 2}$ based four cylinder turbo diesel simulation with air and fuel path simulations suitable for development of control algorithms. It is a mean value model based on phsyical equations which can be fully parametrized. Here, the "out of the box" parameterization was utilized. En-DYNA served as the plant for the GSMMS model and the equations for

\footnotetext{
${ }^{1}$ www.tesis.de

${ }^{2}$ Registered Tradematk of The MathWorks, Natick, MA
} 
the EGR system are discussed here to facilitate an understanding of the complexity of the dynamics. It should be noted that for anomaly detection, isolation and diagnosis, the underlying simulation model was considered unknown, as it would be in a real application.

In the simulation model, the throttle was modeled assuming an isentropic flow [54] as

$$
\begin{aligned}
& \dot{m}_{E G R}=f_{1}\left(\frac{p_{2}}{p_{1}}\right) \cdot f_{2}(\alpha) \cdot f_{3}(T) \cdot f_{4}\left(p_{1}\right) \\
& f_{1}\left(\frac{p_{2}}{p_{1}}\right)=\left\{\begin{array}{cc}
\sqrt{\frac{\left(\frac{p_{2}}{p_{1}}\right)^{\frac{2}{\kappa}}-\left(\frac{p_{2}}{p_{1}}\right)^{\frac{\kappa+1}{\kappa}}}{\left(\frac{2}{\kappa+1}\right)^{\frac{2}{\kappa-1}}-\left(\frac{2}{\kappa+1}\right)^{\frac{\kappa+1}{\kappa-1}}}} & \text { for } \frac{p_{2}}{p_{1}}>\left(\frac{2}{\kappa+1}\right)^{\frac{\kappa}{\kappa-1}} \\
1 & \text { for } \frac{p_{2}}{p_{1}} \leq\left(\frac{2}{\kappa+1}\right)^{\frac{\kappa}{\kappa-1}}
\end{array}\right. \\
& f_{2}(\alpha)=\frac{\dot{m}_{\max }}{2} \cdot(1-\cos (2 \alpha)) \quad \text { for } 0 \leq \alpha \leq \frac{\pi}{2} \\
& f_{3}(T)=\sqrt{\frac{T_{\text {ref }}}{T}} \\
& f_{4}\left(p_{1}\right)=\frac{p_{1}}{p_{\text {ref }}}
\end{aligned}
$$

where $\alpha$ is the throttle valve angle, $p_{1}$ and $p_{2}$ are the pressures before and after the throttle valve respectively, $T$ is the gas temperature before the throttle valve and $\dot{m}_{E G R}$ is the air mass flow. The EGR valve controller consists of two look up tables 

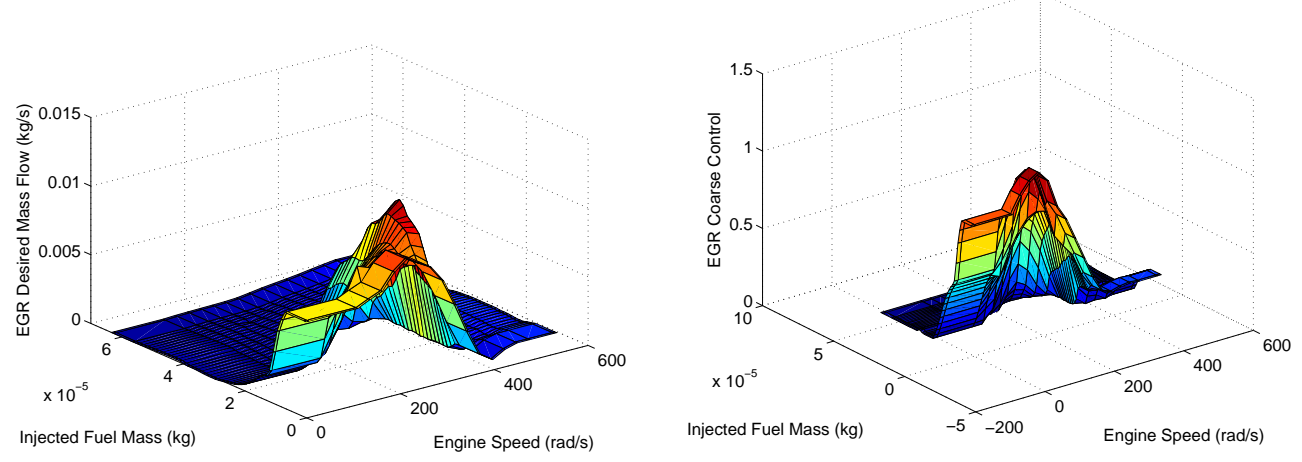

(a) Look up table for the desired EGR mass (b) Look up table for the coarse control for the flow.

EGR valve. A control signal of 1 indicates that the EGR valve is fully open.

which are shown in Fig. 4.3(a) and 4.3(b). These look up tables determine the mass flow $\dot{m}_{\text {desired }}$ and the coarse control for the throttle angle. A proportional-integral (PI) controller is then used to correct the throttle angle such that the desired mass flow is achieved [54]. Clearly, the look up tables add severe static nonlinearities to the EGR system and one would expect that the fixed parameter PI controller will behave differently for various desired EGR mass flows. The look-up table values and PI controller parameters were provided with the default calibration.

For the cooler, enDYNA utilizes the following model

$$
m_{\mathrm{HE}} \cdot c_{\mathrm{p}, \mathrm{HE}} \cdot \frac{\mathrm{d} T_{\mathrm{HE}}}{\mathrm{d} t}=\dot{Q}_{\mathrm{HE} \rightarrow \mathrm{Ambient}}+\dot{m}_{\mathrm{EGR}} \cdot c_{\mathrm{p}, \mathrm{Gas}} \cdot\left(T_{\mathrm{in}}-T_{\mathrm{out}}\right)
$$

where the subscript HE denotes quantities associated with the heat exchanger, $c_{p}$ represents the heat specific heat capacity and $m_{\mathrm{HE}}$ is the mass of the cooler. The outgoing gas is assumed to be at the temperature of the heat exchanger, or

$$
T_{\text {out }}=T_{\mathrm{HE}}
$$


Finally, the heat transfer from the cooler to the ambient is expressed as

$$
\dot{Q}_{\mathrm{HE} \rightarrow \text { Ambient }}=K\left(\dot{m}_{\mathrm{EGR}}\right) \cdot\left(T_{\mathrm{Ambient}}-T_{\mathrm{HE}}\right)
$$

where $K\left(\dot{m}_{\mathrm{EGR}}\right)$ is the heat transfer coefficient as a function of the EGR mass flow. This value is supplied by a look up table [54].

To test the EGR FDD strategy, several anomalies were introduced into the controller, cooler and valve of the EGR system. This was accomplished by modifying some of the characteristic parameters that en-DYNA uses to simulate the engine dynamics.

For the EGR valve fault, the valve characteristic curve was modified as shown in Fig. 4.3 to simulate a low-flow situation (such as an obstruction). The equation for this fault was

$$
f_{2}(\alpha)=\gamma \frac{\dot{m}_{\max }}{2} \cdot(1-\cos (2 \alpha)) \quad \text { for } 0 \leq \alpha \leq \frac{\pi}{2}
$$

where $\gamma=1$ for a normal situation and $\gamma=0.9,0.8,0.6,0.5$ for various fault scenarios. For the cooler faults, the function $K\left(\dot{m}_{\mathrm{EGR}}\right)$ was modified, simulating fouling of the heat exchanger or diminished airflow. The faults introduced were modifications of the slope of $K\left(\dot{m}_{\mathrm{EGR}}\right)$

$$
K\left(\dot{m}_{\mathrm{EGR}}\right)=\beta K_{\text {Normal }}\left(\dot{m}_{\mathrm{EGR}}\right)
$$

where $\beta=[0.9,0.8,0.6,0.5]$ for Faults $1-4$ respectively. These faults are also shown in Fig. 4.4. Both of the aforementioned faults are clearly operating point dependent and will manifest themselves differently for different operating conditions. Finally, 


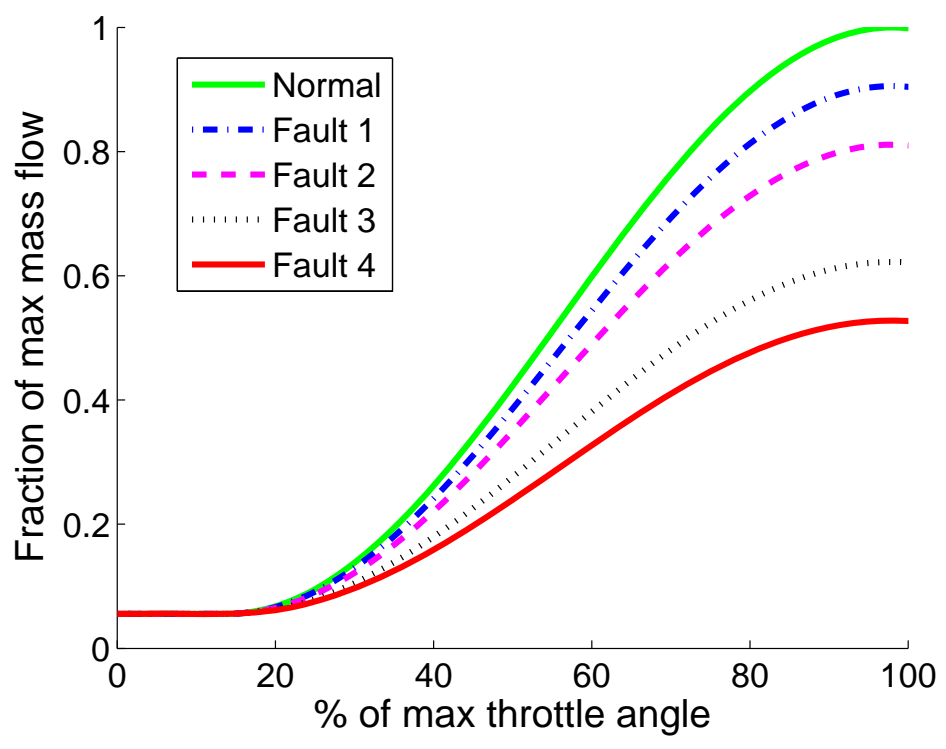

Figure 4.3: Characteristic curves for the EGR valve. These curves are plots of $f_{2}$ in the valve model without (normal) and with four levels of obstruction (10\%, 20\%, $30 \%, 40 \%$ and $50 \%$ ) respectively. 


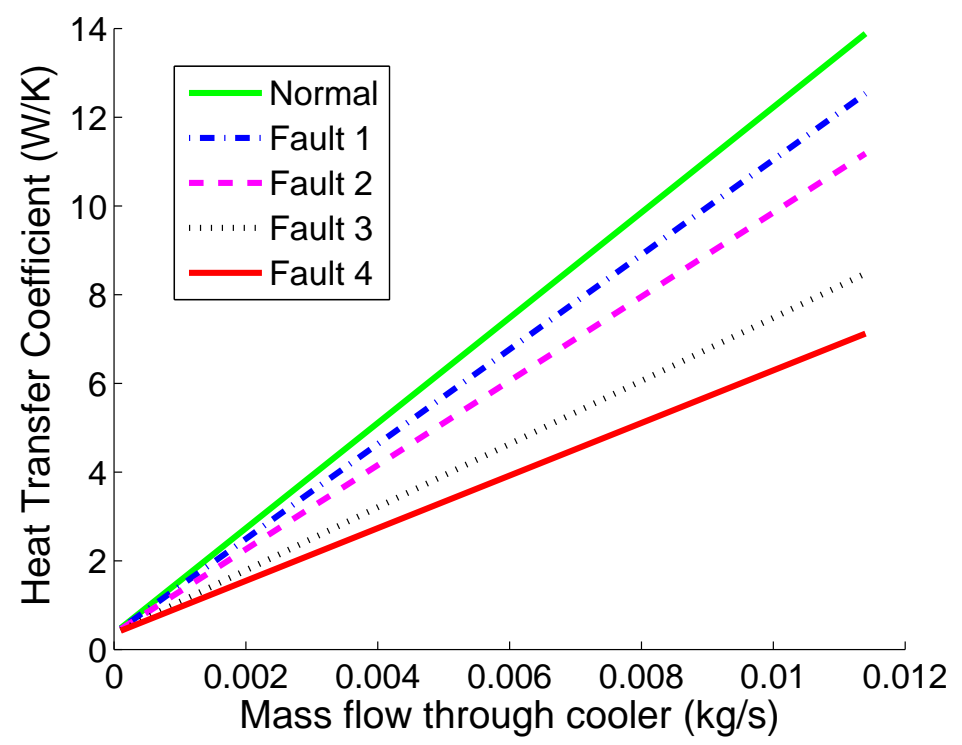

Figure 4.4: $K\left(\dot{m}_{\mathrm{EGR}}\right)$ for the EGR cooler for normal operation and four levels of fouling. As the fouling increases, the heat transfer coefficient, $\mathrm{K}$, decreases.

a controller fault was simulated as a delay between the PI controller and the throttle angle, as depicted in Fig. 4.5. This delay emulates a scenario where the ECU communication is degraded. The variable $d$ is the number of samples of the delay. The controller delays of $10 \mathrm{~ms}, 15 \mathrm{~ms}$, and $20 \mathrm{~ms}$ were introduced.

\subsection{EGR Diagnostic System Design}

Examining the block diagram in Fig. 4.2, it can be seen that the EGR system can be decomposed into a mass flow and temperature subsystem, as in Fig. 4.6. However, this decomposition is not complete since the EGR mass flow determines the heat transfer characteristics of the cooler (Eq. 4.2). Therefore, at the top level, two anomaly detectors are required, one to monitor the mass flow subsystem 


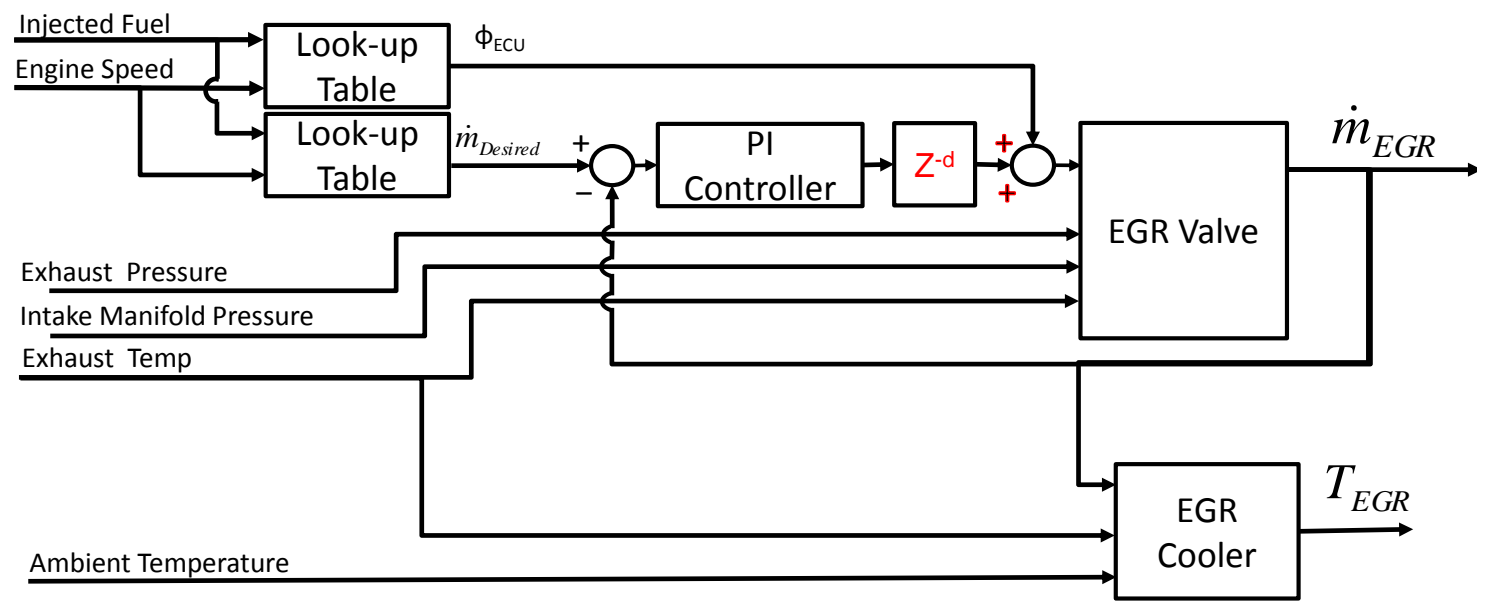

Figure 4.5: Delay faults introduced into the EGR controller.

and one to monitor the cooler subsystem as depicted in Fig. 4.7. This strategy decomposes the fault detection task into two smaller, decoupled subsystems. By monitoring the CVs of each $\mathrm{AD}$, a fault can be detected and isolated to the mass flow or cooler subsystem. Furthermore, if the mass flow system is determined to be faulty, additional ADs for each of the constituent mass flow subsystems can be monitored, as illustrated in Fig. 4.8. Only the ADs monitoring faulty subsystems will signal anomalies, thus facilitating isolation of the subsystem(s) causing the anomalous behavior.

\subsection{Training the GSMMS Models}

En-DYNA was used to generate engine data and the signals were polluted with $2 \%$ additive noise after the simulation to emulate sensor noise. For each of the blocks in 4.2, a GSMMS for normal behavior was constructed. Three standard 


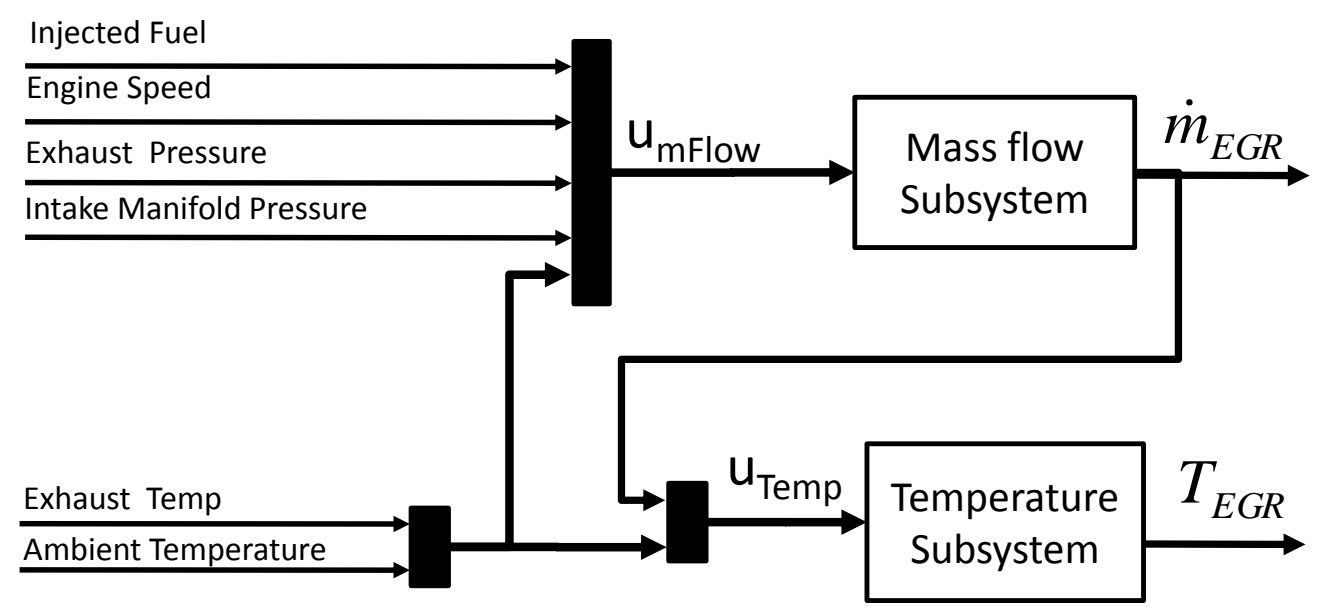

Figure 4.6: Simplified block diagram of the EGR system into a mass flow and temperature subsystem.

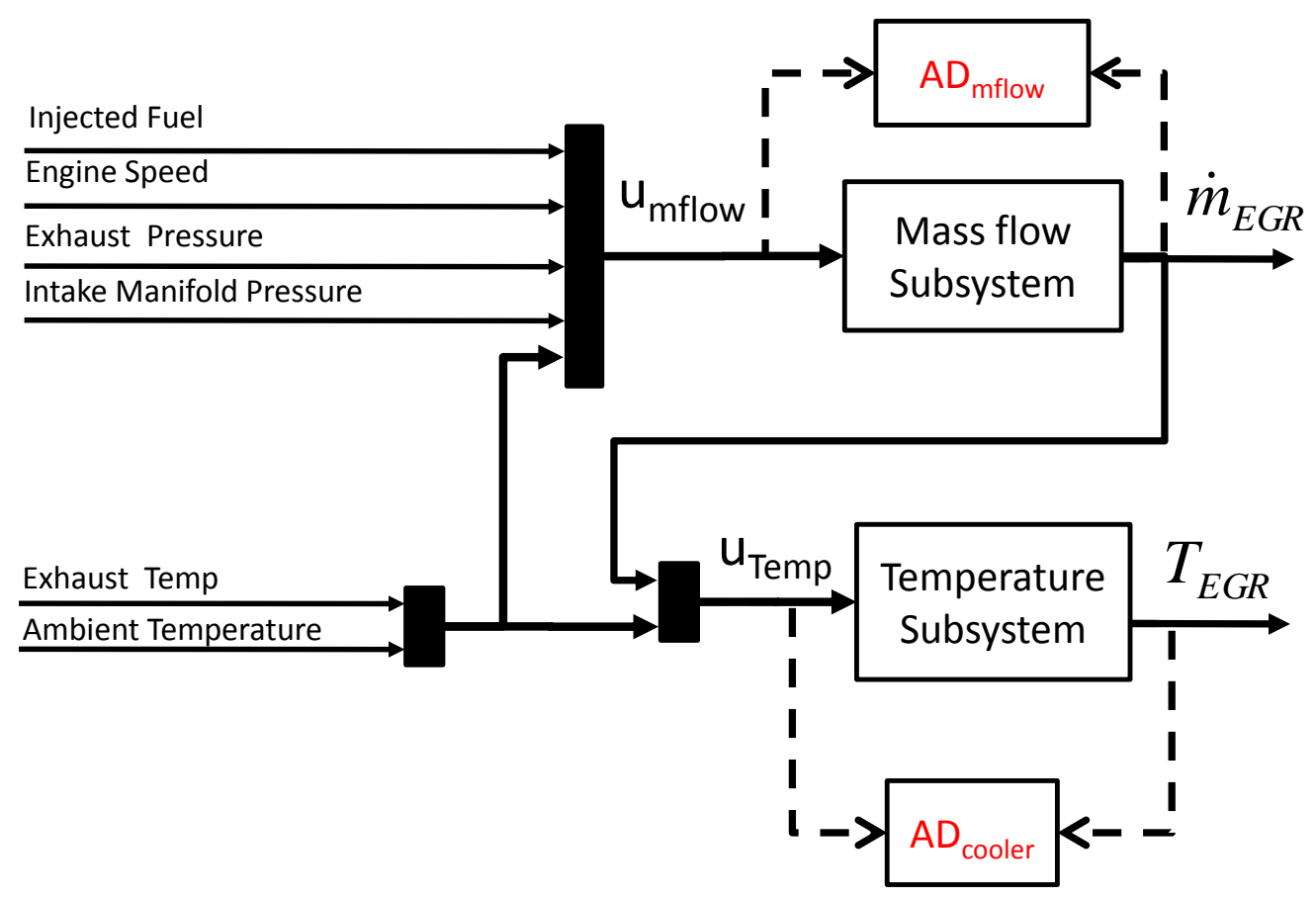

Figure 4.7: Top level anomaly detection strategy for the EGR System. 


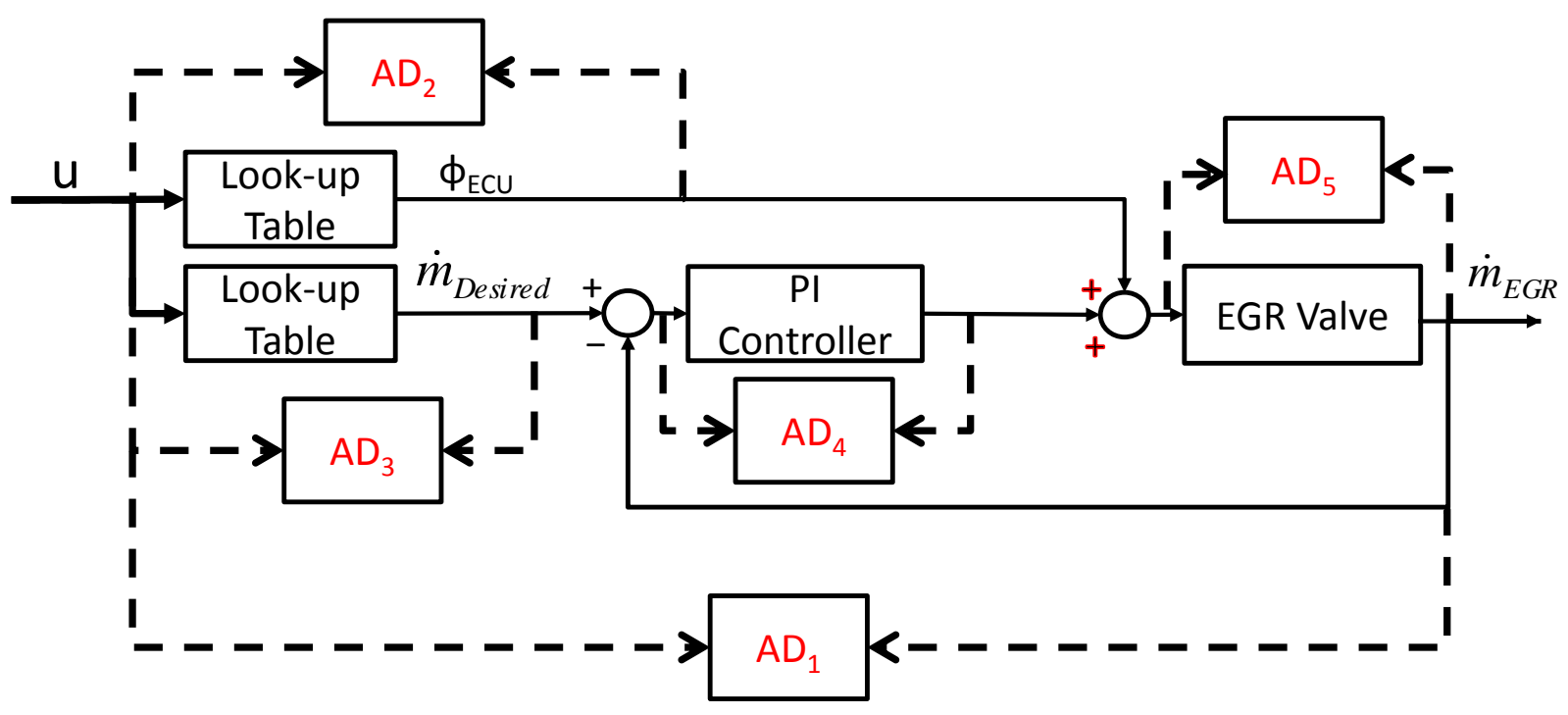

Figure 4.8: Fault Isolation in the EGR mass flow subsystem using distributed anomaly detection.

driving profiles (ECE-15, FTP-75 and MVEG-B) were used to generate training data and a fourth profile (Japan 10-15) was used to test the generalization capability of the model. The trainig and testing data velocity profiles are shown in Fig. 4.9. During training, the ambient tempeature was varied between $10^{\circ}-30^{\circ} \mathrm{C}$ to account for different ambient conditons. The GSMMS models of normal and faulty behavior modes were trained offline using inputs and outputs from relevant subsystems. The training procedure followed the batch algorithm detailed in Section 3.1.2, with the stopping criteria being a maximum SOM size. For each GSMMS, the appropriate inputs were selected and autoregressive orders of the local models were provided. For this work, the maximum number of regions and orders of the local models were determined in an ad-hoc manner. First, the max SOM size was increased until there was no further improvement in the global RMS error. After this, the 


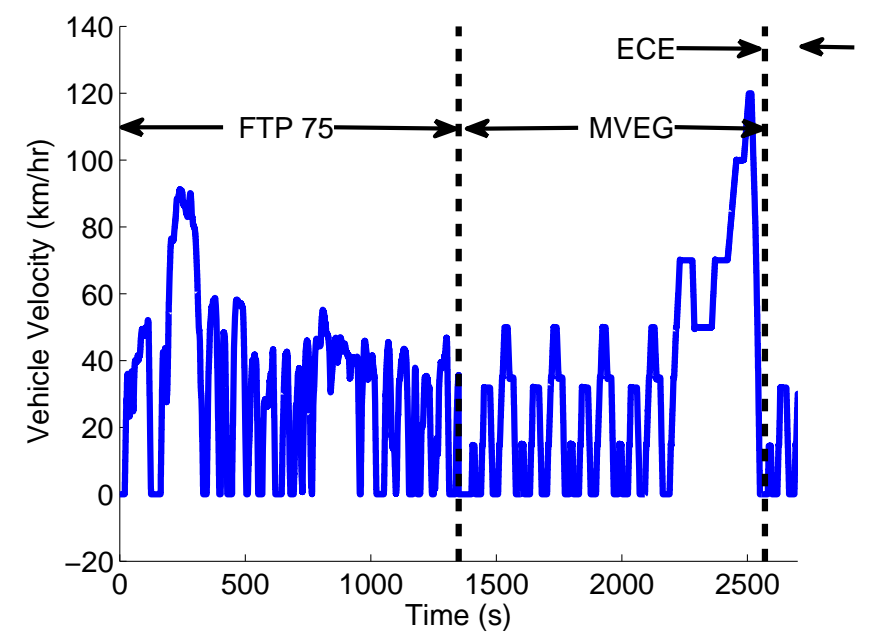

(a) Driving profile utilized for training the GSMMS models. The profile is a concatenation of three standard driving profiles: FTP 75, MVEG-B, and ECE.

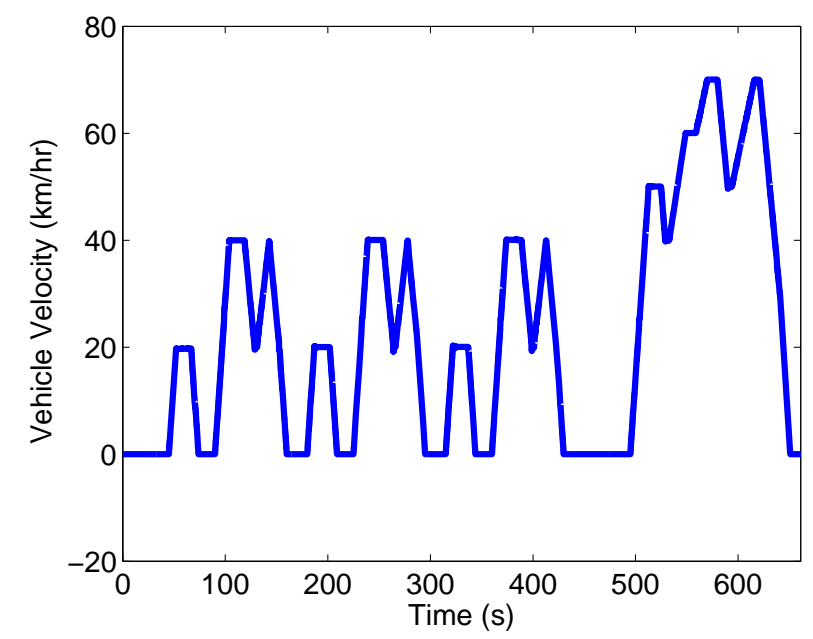

(b) Japan 10-15 driving profile utilized for testing the GSMMS models. The profile is not used for training.

Figure 4.9: Driving profiles utilized for training and testing of the GSMMS models. 
regional residuals were visually examined for whiteness and the local order were increased until improvements to the residual whiteness were not longer evident. For this study, no formal criteria (such as F-tests [55] or Minimum Description Length [56]) was used. While not used in this study, recent work has shown that input selection, model orders and the number of regions can be automated [57] using the Minimum Description Length criterion as a test of adequacy.

A few additional modifications were used for this application. It was found during the modeling procedure that inserting nodes based solely on root-mean square error, as was done in [44], resulted in some regions with high visitation frequencies where the residuals did not follow a Gaussian Distribution. This indicated a possibly poor approximation since local model parameters were estimated using a least squares approach. An example of such a situation is shown in Fig. 4.10, where the majority of the data conforms to a single model, but a small "tail" does not conform well. In this case, the root-mean square residuals may be low, but the region at the end of the data will be modeled poorly. Clearly, if one inserts an additional linear model (and allows the SOM to update for a few epochs) a second model can represent the data better, as shown in Fig. 4.11). To improve modeling accuracy and push modeling errors to follow a Gaussian distribution, a new node was inserted between the region $m$ satisfying

$$
\max _{m} \operatorname{card}\left\{e_{m}:\left|e_{m}\right|>4 \sigma_{m}\right\}
$$

and its furthest neighbor (in Euclidean distance). In the above equation, card $\{\cdot\}$ denotes the set cardinality, $e_{m}$ are the modeling errors and $\sigma_{m}$ is the standard deviation 


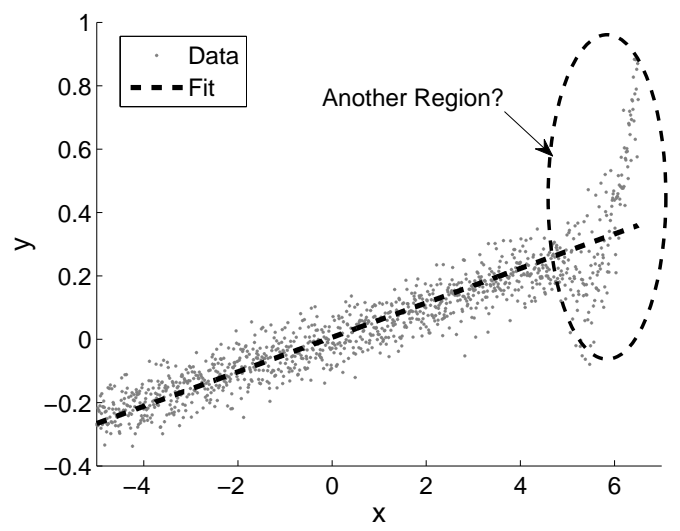

(a) Single linear model fit to data

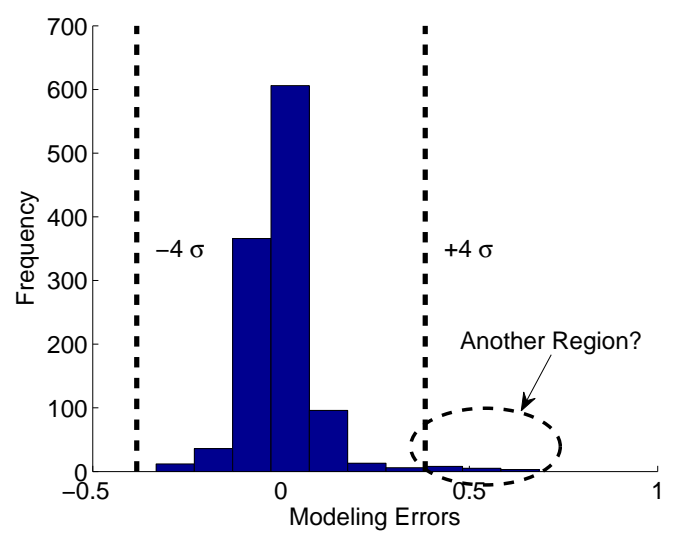

(b) Histogram of residuals with $4 \sigma$ limits

Figure 4.10: Example of a situation where a majority of the data is well represented by a single linear model, but a small region $(x>5.5)$ is modeled poorly. In the histogram, one sees that a result of the poor fit is a skewed residual distribution, leading to a significant number of points outside of the $4 \sigma$ limits. 

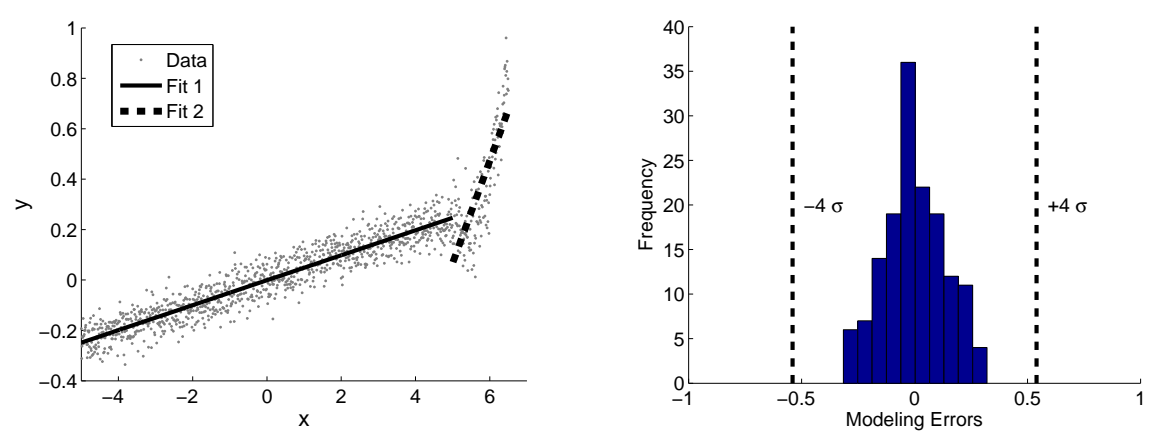

(a) Two linear models fit to the same data (b) Histogram of model 1 residuals with 4 as in Fig. 4.10 $\sigma$ limits

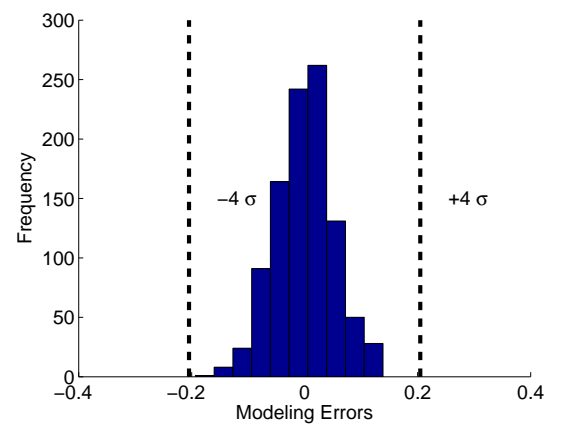

(c) Histogram of model 2 residuals with 4 $\sigma$ limits

Figure 4.11: Using two models, the for $x>5.5$ is represented better and the residual histograms appear more Gaussian than with one model. This idea was the inspiration for the new node insertion criteria (Eq. 4.5). 
of the errors for region $m$. Since the $\pm 4 \sigma$ limits contain over $99.99 \%$ of the data for a normal distribution, the above criteria can be considered a measure of local residual non-normality. This additional step is enclosed in a dashed line in Fig. 4.12.

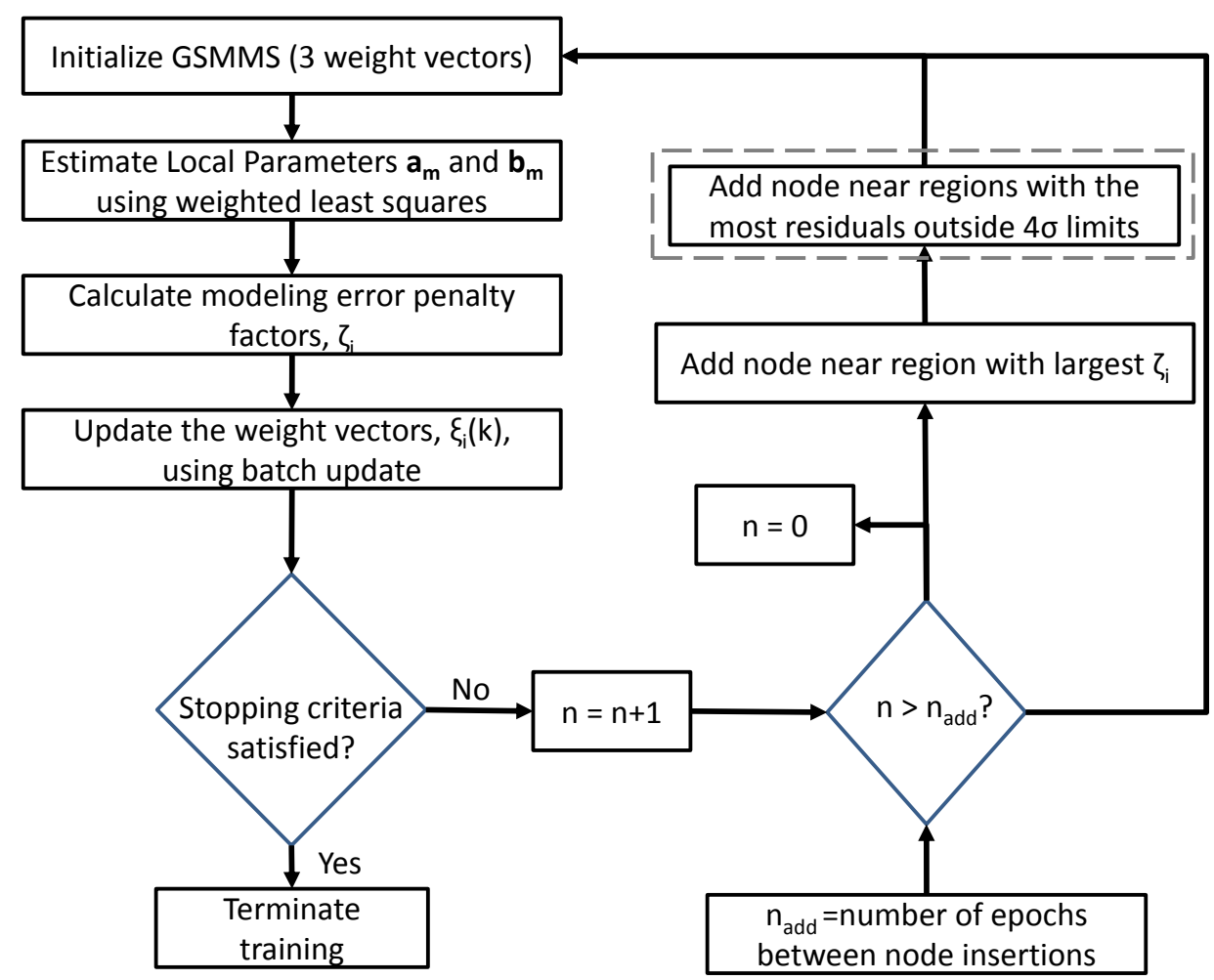

Figure 4.12: Modification to GSMMS training procedure.

The modeling performance of the GSMMS approach can be seen in Fig. 4.13 and 4.14 for the overall anomaly detectors illustrated in Fig. 4.7. The overall anomaly detectors represent the most complex situation since the look-up tables are included and the closed loop dynamics of the controller and plant must be ac- 
counted for. A summary of the GSMMS models for each subsystem can be seen in Tables 4.1, 4.2 and 4.3.

\begin{tabular}{|c|c|c|}
\hline Subsystem & Inputs & Outputs \\
\hline Mass flow subsystem & $\begin{array}{c}\text { Injected Fuel Mass, Engine Speed, } \\
\text { Exhaust Temp,Exhaust Pressure, } \\
\text { Intake Manifold Pressure, } \\
\text { Ambient Temp }\end{array}$ & $\begin{array}{c}\text { EGR } \\
\text { Mass Flow }\end{array}$ \\
\hline Cooler subsystem & $\begin{array}{c}\text { EGR Mass Flow, Ambient Temp, } \\
\text { Exhaust Temp }\end{array}$ & $\begin{array}{c}\text { EGR } \\
\text { Gas Temp }\end{array}$ \\
\hline EGR valve & $\begin{array}{l}\text { EGR Angle, Exhaust Temp, } \\
\text { Exhaust Pressure, } \\
\text { Intake Manifold Pressure, } \\
\text { Ambient Temp }\end{array}$ & $\begin{array}{c}\text { EGR } \\
\text { Mass Flow }\end{array}$ \\
\hline PI controller & $\begin{array}{l}\text { EGR Mass Flow Error, } \\
\text { EGR Coarse Control }\end{array}$ & $\begin{array}{l}\text { EGR } \\
\text { Angle }\end{array}$ \\
\hline Mass flow look-up table & $\begin{array}{l}\text { Injected Fuel Mass, } \\
\text { Engine Speed }\end{array}$ & $\begin{array}{l}\text { Desired } \\
\text { Mass Flow }\end{array}$ \\
\hline Coarse control look-up & $\begin{array}{l}\text { Injected Fuel Mass, } \\
\text { Engine Speed }\end{array}$ & $\begin{array}{c}\text { Coarse } \\
\text { EGR Angle }\end{array}$ \\
\hline
\end{tabular}

Table 4.1: Inputs for the various GSMMS models. 

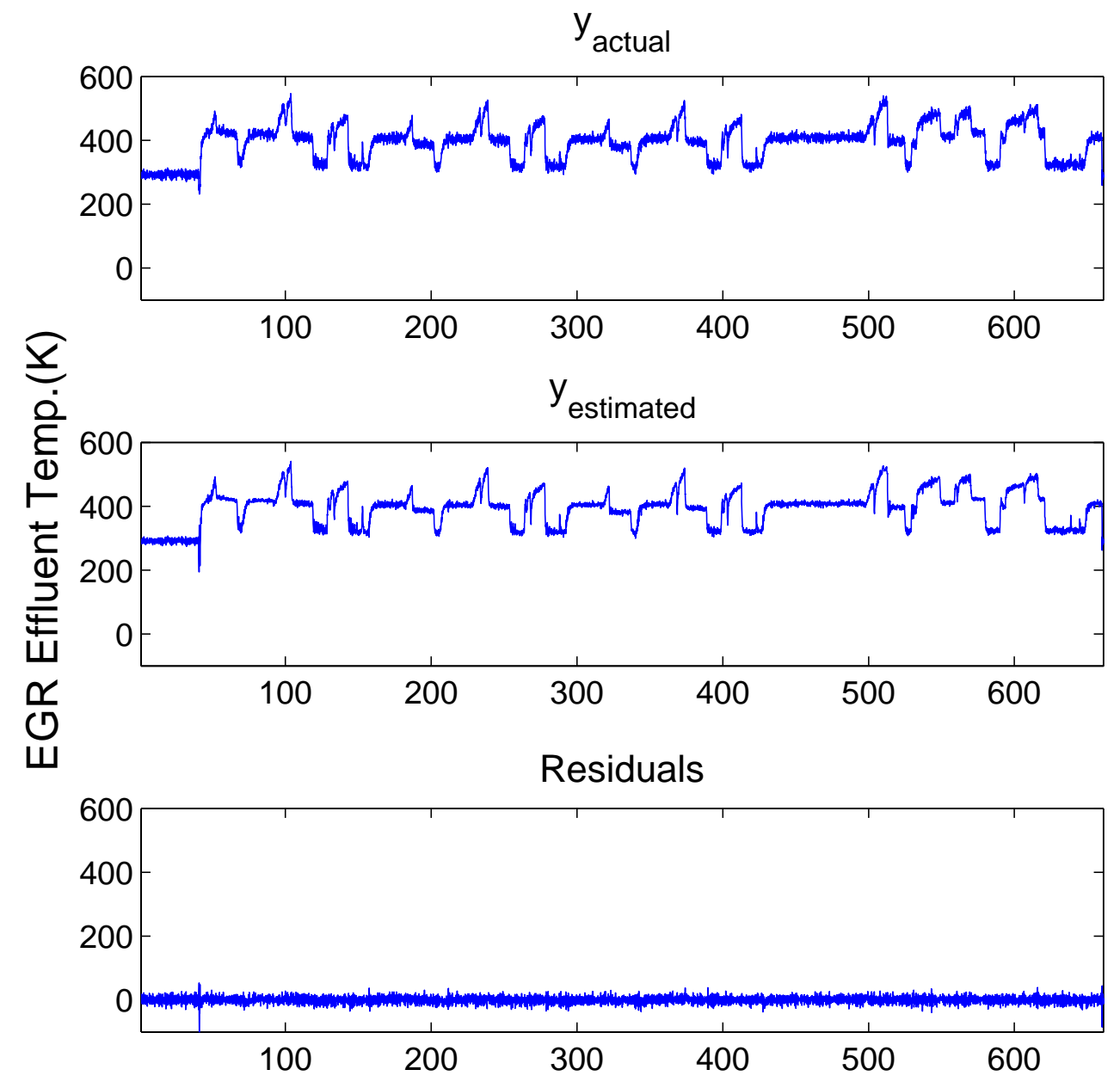

Figure 4.13: Modeling Performance of the GSMMS for $\mathrm{AD}_{\text {cooler }}$ without a fault. The system was exercised with a standard driving profile (Japan 10-15) that was not utilized for training. 


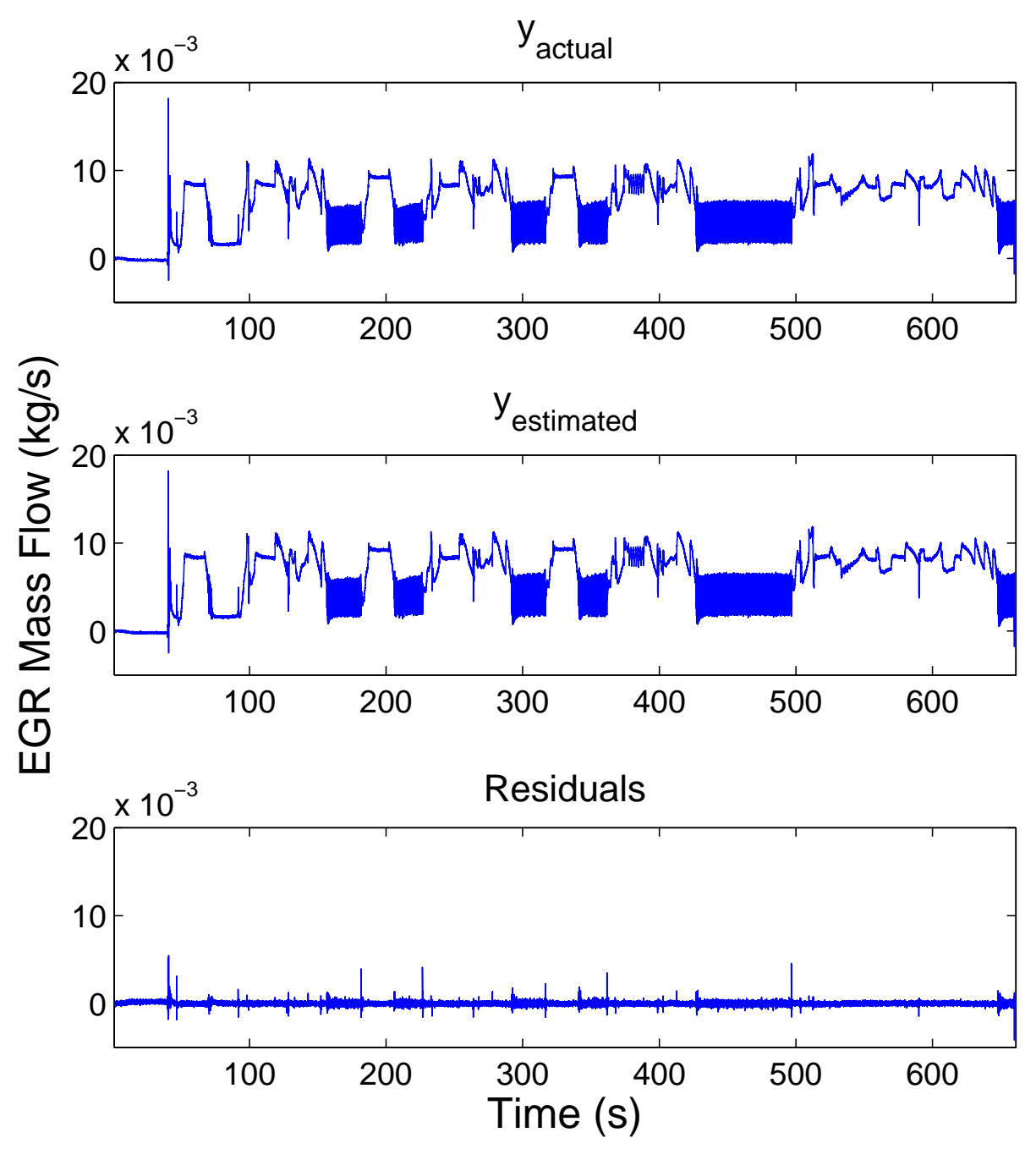

Figure 4.14: Modeling Performance of the GSMMS for $\mathrm{AD}_{\text {valve }}$ without a fault. The system was exercised with a standard driving profile (Japan 10-15) that was not utilized for training. 


\begin{tabular}{c|c|c|c|c} 
Subsystem & Regions & $\mathbf{n}_{\mathbf{a}}$ & $\mathbf{n}_{\mathbf{b}}$ & Output Range \\
\hline Mass flow subsystem & 39 & 2 & {$[2,2,2,2,2,1]$} & $0-1.9 \cdot 10^{-2} \mathrm{~kg} / \mathrm{s}$ \\
Cooler subsystem & 21 & 1 & {$[1,1,1]$} & $270-800 \mathrm{~K}$ \\
EGR Valve & 41 & 2 & {$[2,2,2,2,1]$} & $0-1.9 \cdot 10^{-2} \mathrm{~kg} / \mathrm{s}$ \\
PI Controller & 1 & 5 & {$[5,5]$} & $0-90^{\circ}$ \\
Mass Flow look-up & 35 & 0 & {$[1,1]$} & $2.1 \cdot 10^{-4}-1.1 \cdot 10^{-2}$ \\
Coarse Angle look-up & 35 & 0 & {$[1,1]$} & $0-1$
\end{tabular}

Table 4.2: Model summary for the GSMMS models trained for anomaly detection.

\begin{tabular}{c|c} 
Subsystem & RMS error \\
\hline Mass flow subsystem & $1.7 \cdot 10^{-4} \mathrm{~kg} / \mathrm{s}$ \\
Cooler subsystem & $10.4 \mathrm{~K}$ \\
EGR Valve & $1.7 \cdot 10^{-4} \mathrm{~kg} / \mathrm{s}$ \\
PI Controller & $2.3^{\circ}$ \\
Mass Flow look-up & $5.1 \cdot 10^{-4} \mathrm{~kg} / \mathrm{s}$ \\
Coarse Angle look-up & $2.8 \cdot 10^{-2}$
\end{tabular}

Table 4.3: RMS errors of normal models computed over 661 seconds of a profile that was not used for training. 


\subsection{Fault Detection and Isolation}

During operation, the CVs for the top-level anomaly detection (see Fig. 4.7) are monitored. If $\mathrm{AD}_{\text {mflow }}$ flags an anomaly, the EGR mass flow subsystem anomaly detectors in Fig. 4.8 are monitored to isolate the fault to one of the constituent subsystems. If $\mathrm{AD}_{\text {cooler }}$ flags an anomaly, the fault is isolated to the EGR cooler subsystem. This AD hierarchy is summarized in Fig. 4.15. Unlike the previous research in automotive air path fault detection, no fault models or faulty data is used to detect or isolate faults.

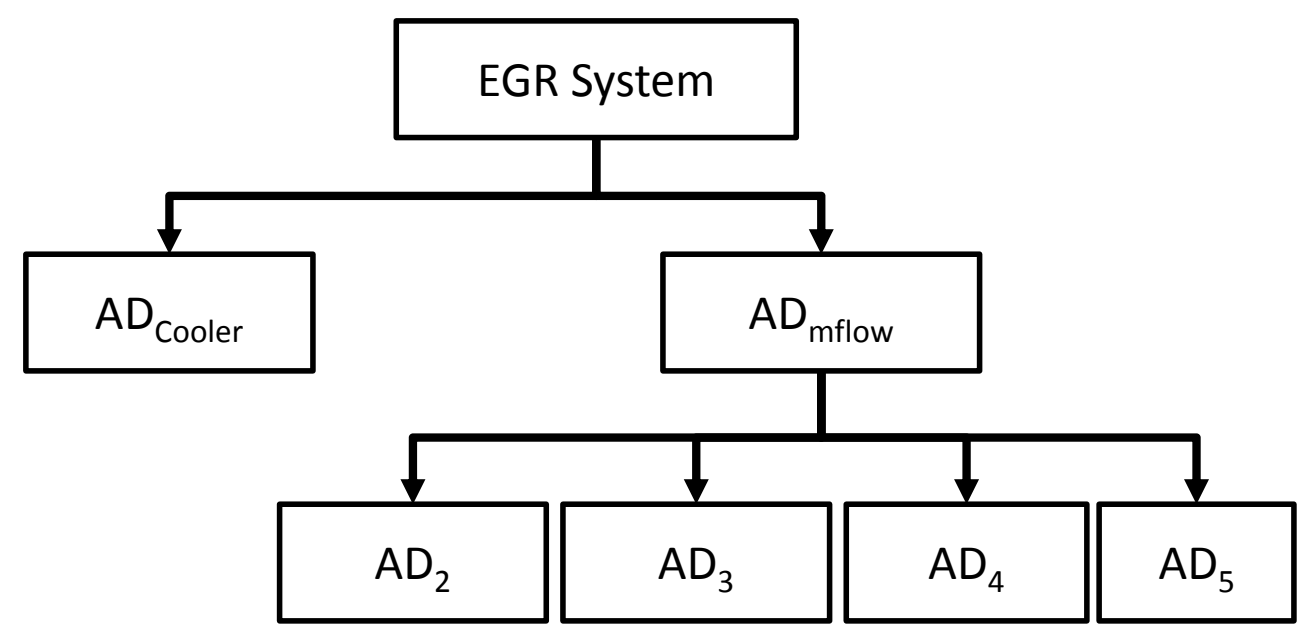

Figure 4.15: Anomaly Detector hierarchy. ADs 2-5 are only monitored if the $\mathrm{AD}_{\text {mflow }} \mathrm{CV}$ is low.

In this section, the faults presented in Section 4.1 are detected and isolated using the GSMMS strategy. Both global and local CVs are examined and any practical issues regarding the anomaly detection are discussed. In all cases the faults were introduced at 1322 seconds ( 2 cycles of the normal test data) so that the nor- 
mal CV evolution could be compared to the faulty behavior.

\subsubsection{EGR Valve Faults}

Examining Fig. 4.7 and 4.8, it is clearly expected that CVs output by the $\mathrm{AD}_{\text {mflow }}$ should decrease while $\mathrm{AD}_{\text {cooler }}$ should remain near 1. Figure 4.16 shows the top level anomaly detection CVs. As expected, the $\mathrm{AD}_{\text {mflow }}$ indicates an anomaly for the various faults that were inserted except for the smallest fault (Fault 1, 10\%

fouling). This is due to the fact that $\mathrm{AD}_{\text {mflow }}$ is monitoring the closed-loop mass flow subsystem and the PI controller is able to mitigatethe effects of a small obstruction. The isolation of the fault can then be accomplished by interpreting CVs output by $\mathrm{AD}_{2-5}$ monitoring the mass flow subsystem. As expected, only $\mathrm{CV}$ s output by $\mathrm{AD}_{5}$ drop for the inserted faults, as can be seen in Fig. 4.8. Note that $\mathrm{AD}_{5}$ is much more sensitive to the obstruction fault since the controller is no longer included in the input-output mapping.

\subsubsection{Cooler Fault}

For the cooler faults, it is expected that only $\mathrm{AD}_{\text {cooler }}$ will signal an anomaly and that $\mathrm{AD}_{\text {mflow }}$ will remain near 1 . Figure 4.18 show the $\mathrm{CV}$ s for the top level ADs and Fig. 4.19 shows the CVs for the various mass flow subsystems. The mass flow ADs are shown as a "sanity check" and are not needed for isolation. The global $\mathrm{CV}$ for $\mathrm{AD}_{\text {cooler }}$ does not appear to be that sensitive to the smaller cooler faults. As mentioned in Section 3.2, the global CV defined by Eq. 3.14 is intended to simplify the anomaly detection by allowing one to monitor one value for each 


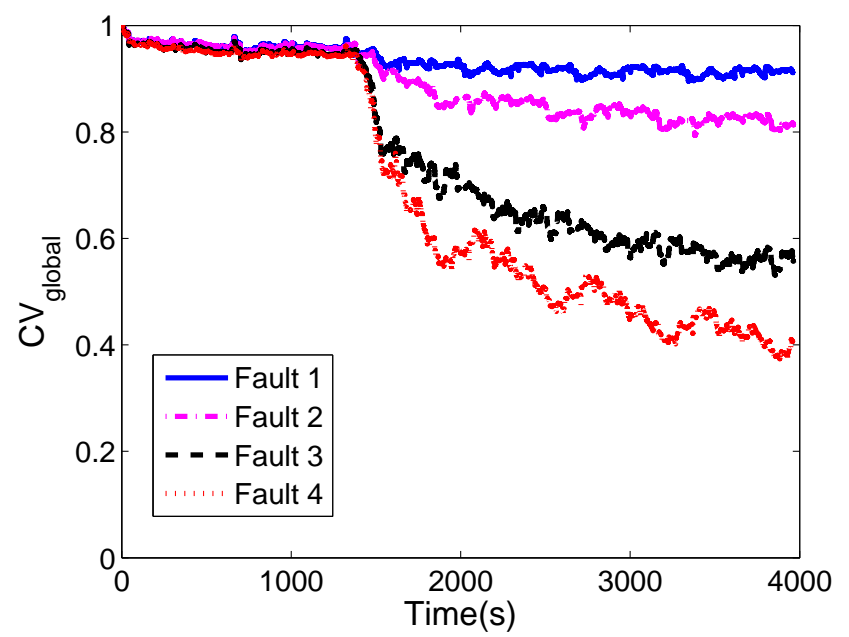

(a) $\mathrm{AD}_{\text {mflow }}$

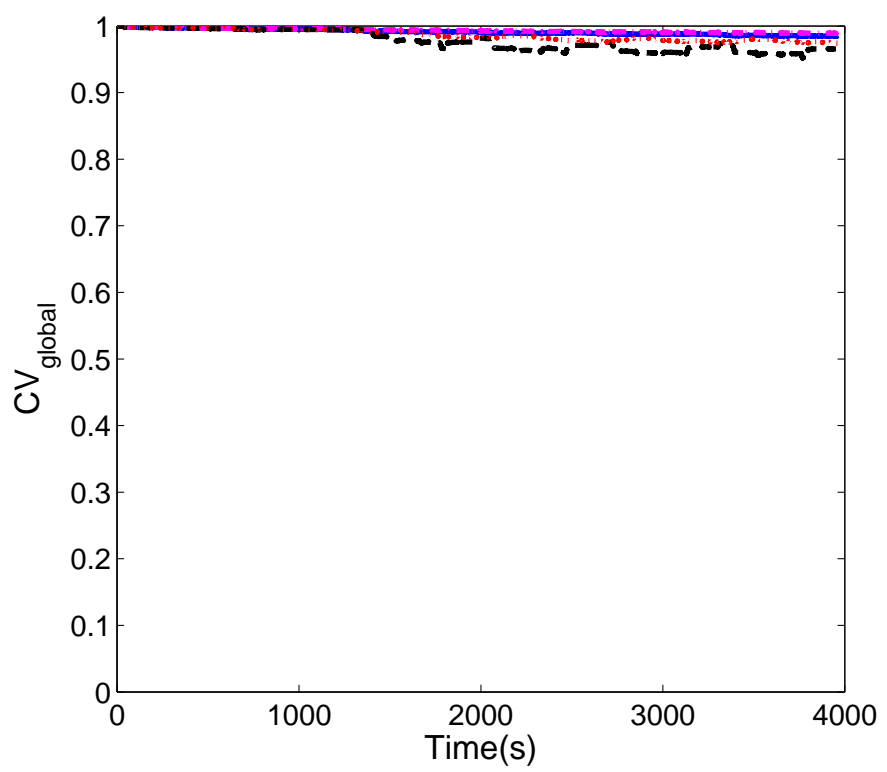

(b) $\mathrm{AD}_{\text {cooler }}$

Figure 4.16: $\mathrm{CV}_{\text {global }}$ for the top level anomaly detectors. A valve anomaly introduced at 1322 seconds. Fault 1 is difficult to distinguish since since the $\mathrm{CV}$ change is small compared to the normal system. This is expected since $\mathrm{AD}_{\text {mflow }}$ is monitoring the closed-loop system and the controller is able to compensate for small faults. 


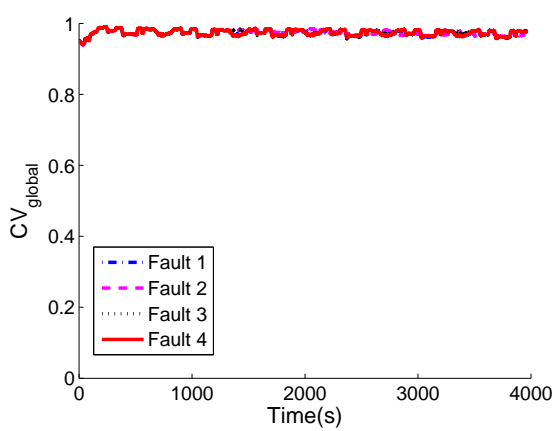

(a) $\mathrm{AD}_{2}$

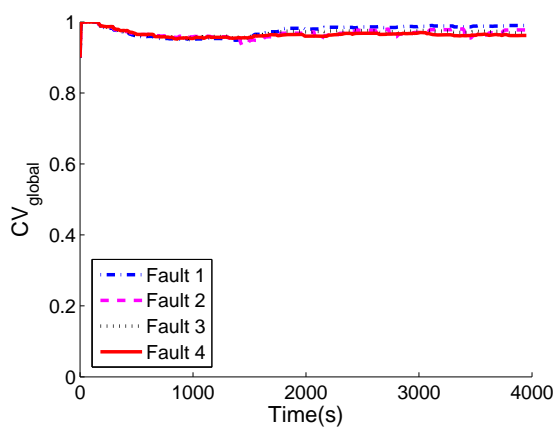

(c) $\mathrm{AD}_{4}$

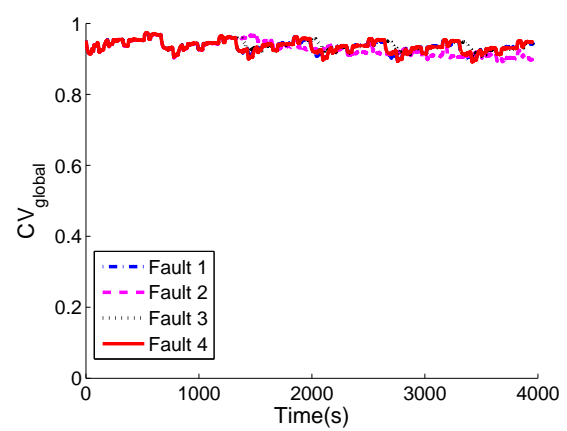

(b) $\mathrm{AD}_{3}$

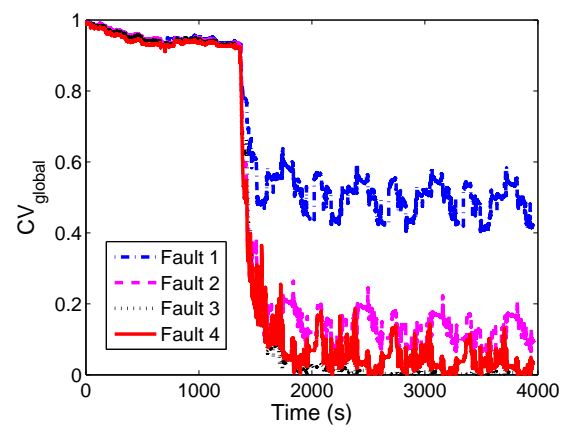

(d) $\mathrm{AD}_{5}$

Figure 4.17: Fault isolation using distributed anomaly detectors for a valve anomaly introduced at 1322 seconds. 


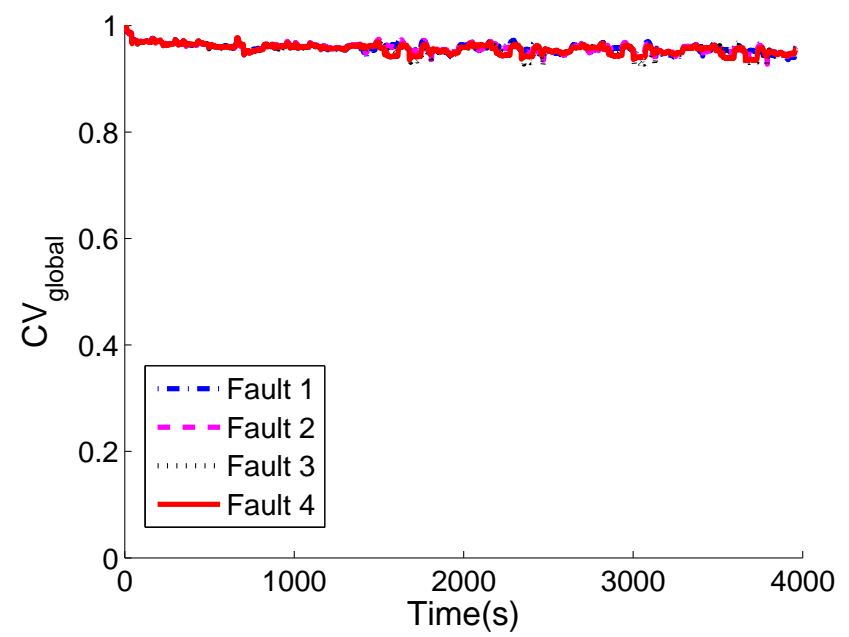

(a) $\mathrm{AD}_{\text {mflow }}$

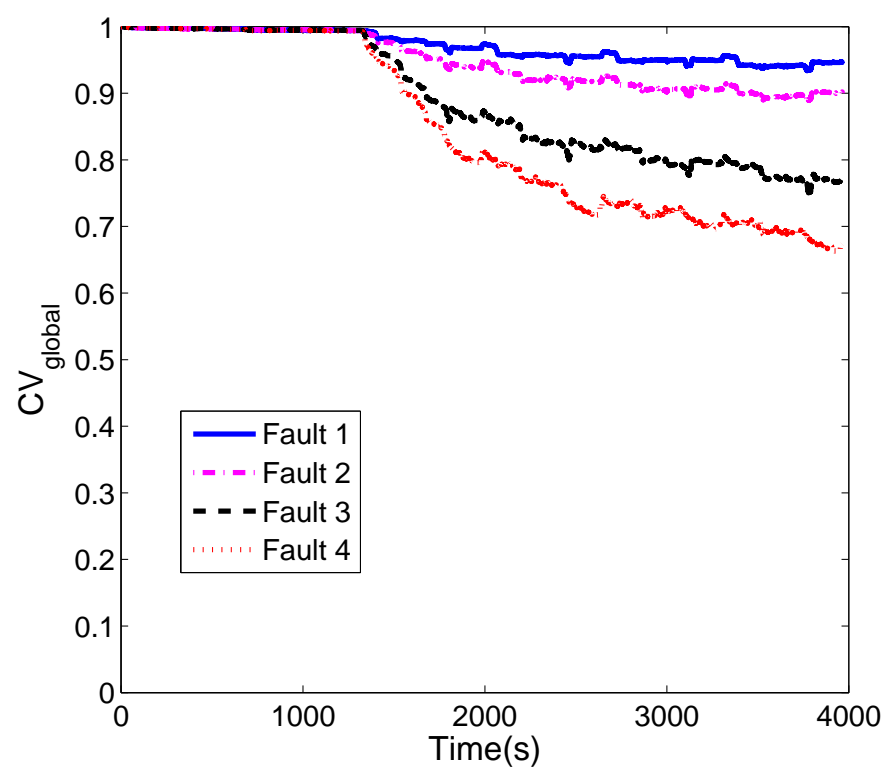

(b) $\mathrm{AD}_{\text {cooler }}$

Figure 4.18: $\mathrm{CV}_{\text {global }}$ for the top level anomaly detectors a cooler anomaly introduced at 1322 seconds. As expected, the cooler AD CV is low. 


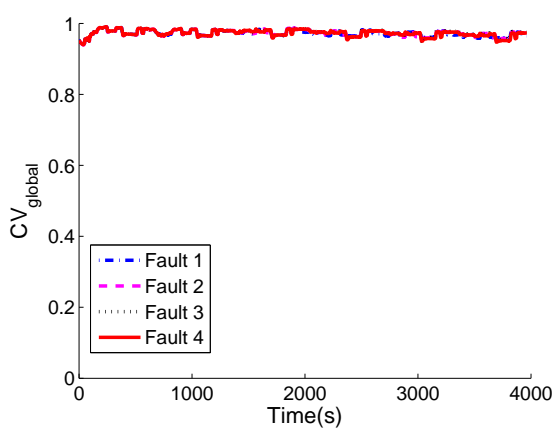

(a) $\mathrm{AD}_{2}$

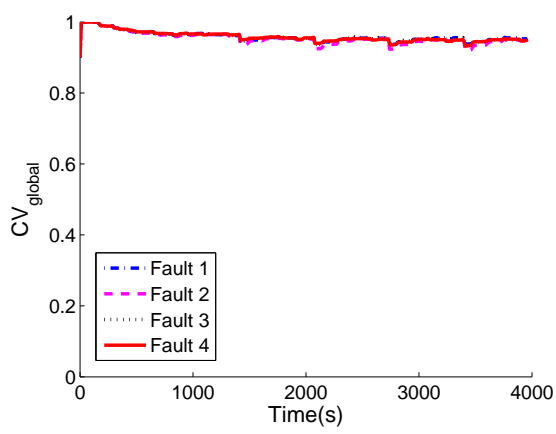

(c) $\mathrm{AD}_{4}$

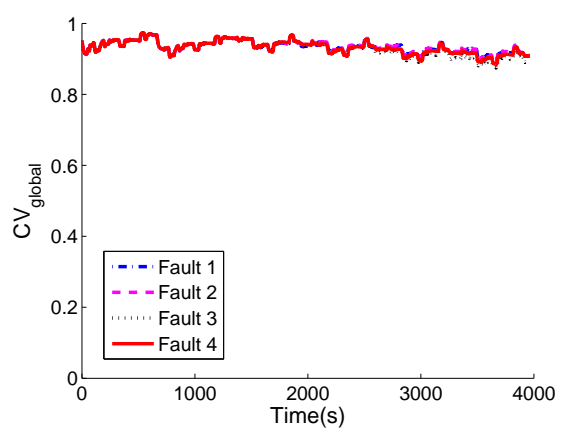

(b) $\mathrm{AD}_{3}$

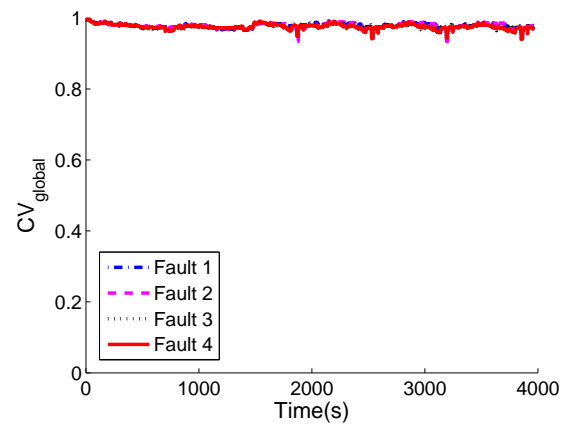

(d) $\mathrm{AD}_{5}$

Figure 4.19: Mass flow subsystem CVs for a cooler anomaly. As expected, all of these CVs are near 1 . 
AD. However, it is typical for faults to manifest themselves differently in different regions of the state space[11,22] and thus the local CVs will behave differently. With this in mind, one low CV is enough to signal an anomaly. Thus, one can look at each of the $M$ regional CVs to determine if the behavior is anomalous. Figure 4.20 shows two local CVs under Fault 1 (least severe) conditions. The two CVs selected for this plot (out of a possible 61) were the most and least sensitive. Thus, the data plotted here are the two extremes in the global CV averaging. Similarly, the extrmeme local CVs for the more severe faults are plotted in Fig. 4.21, 4.22 and 4.23. Clearly, the regional CVs are indicating anomalous behavior, even in the case of Fault 1, where $C V_{\text {global }}$ is still high (see Fig. 4.18).

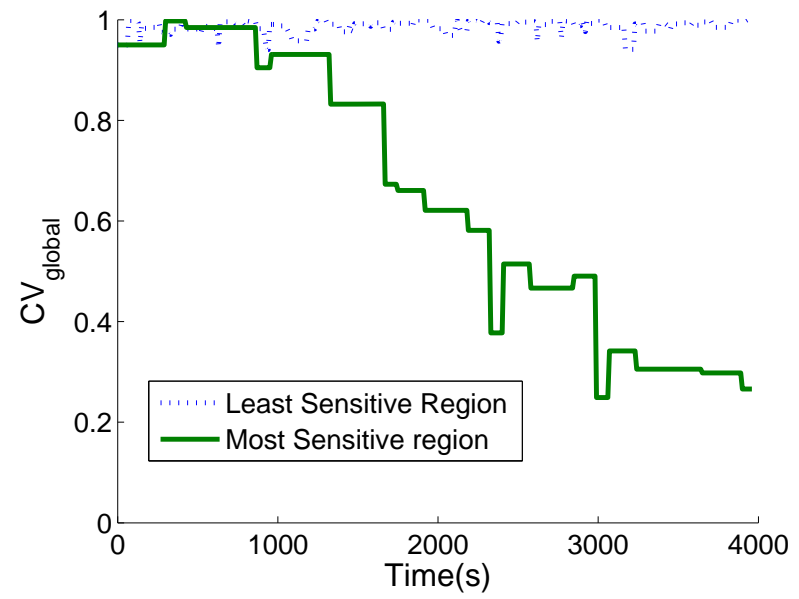

Figure 4.20: Extreme regional confidence values for $A D_{\text {cooler }}$ for Fault 1. The CVs that drop the most (and least) during testing are shown. The remaining regional $\mathrm{CVs}$ are within these local extremes. 


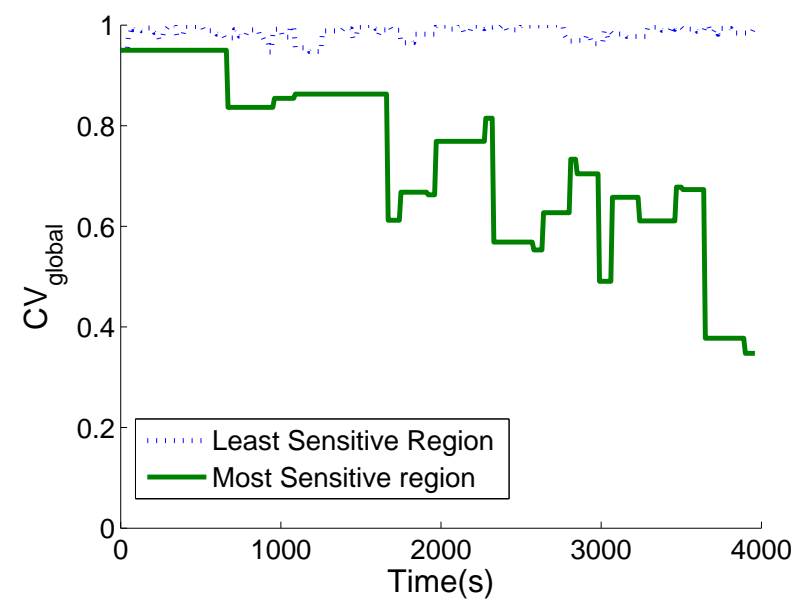

Figure 4.21: Local extreme confidence values for $\mathrm{AD}_{\text {cooler }}$ for Fault 2. The CVs that drop the most (and least) during testing are shown. The remaining regional $\mathrm{CV}$ s are within these local extremes.

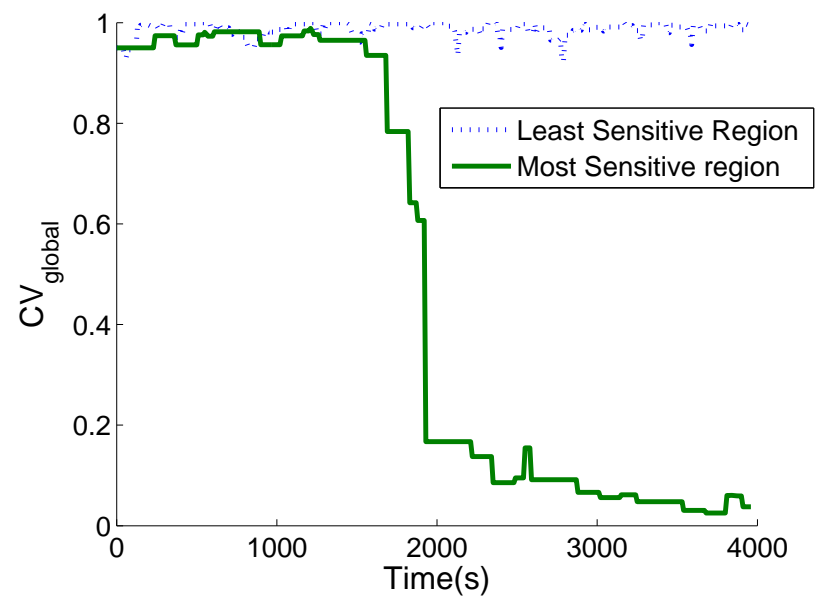

Figure 4.22: Local extreme confidence values for $\mathrm{AD}_{\text {cooler }}$ for Fault 3. The CVs that drop the most (and least) during testing are shown. The remaining regional CVs are within these local extremes. 


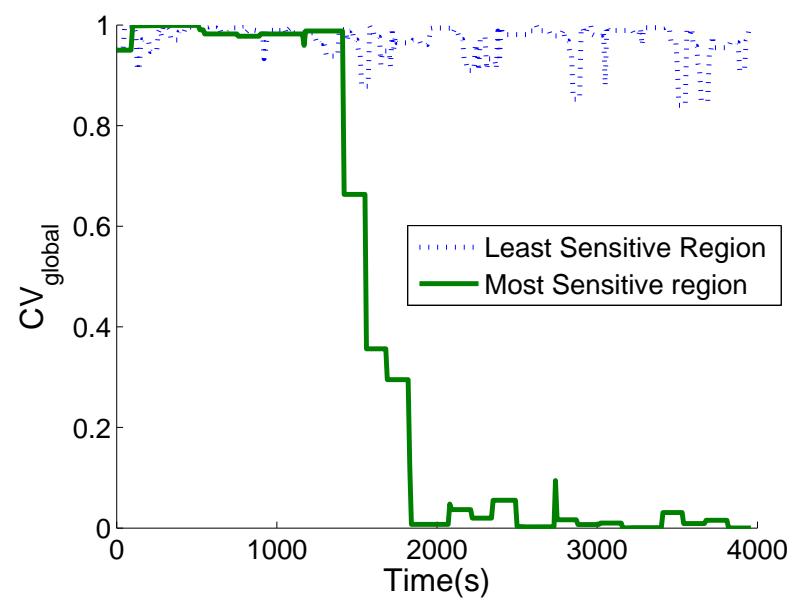

Figure 4.23: Local extreme confidence values for Fault $\mathrm{AD}_{\text {cooler }}$ for 4 . The $\mathrm{CV}$ s that drop the most (and least) during testing are shown. The remaining regional $\mathrm{CVs}$ are within these local extremes.

\subsubsection{Controller Fault}

The final fault that was introduced was a delay after the PI controller, as illustrated in Fig. 4.5. Introduction of the delay yields a controller transfer function of the form

$$
Y_{\text {delayed }}(s)=e^{-s T} C(s) E(s)
$$

where $C(s)$ is the original controller transfer function, $E(s)$ is the Laplace Transform of the input and $T$ is the delay. Applying a Final Value Theorem with a step change in $E(s)[58]$ yields

$$
\lim _{t \rightarrow \infty} y_{\text {delayed }}(t)=\lim _{s \rightarrow 0} Y_{\text {delayed }}(s)=C(0)=\lim _{t \rightarrow \infty} y(t)
$$

where $y_{\text {delayed }}(t)$ and $y(t)$ are the inverse Laplace Transforms of $Y_{\text {delayed }}(s)$ and $\left.Y_{(} s\right)$ respectively. Thus, the behavior of the nominal system $(y(t))$ and the delayed system $\left(y_{\text {delayed }}(t)\right)$ will be identical in the steady-state. Following Liu et al. [59] an 
excitation requirement was placed on the output so that only the transient information is utilized in anomaly detection. Clearly, if the output variance is low, the system is near steady-state and transient faults will not manifest themselves. For this reason, the local CVs in the PI controller were updated only when

$$
\operatorname{Var}[y(k-n a), y(k-n a+1), \ldots, y(k)]>\epsilon
$$

where $\epsilon$ was a small positive constant. For the PI ADs, $\epsilon=3.24$ which represented a standard deviation of $1.8^{\circ}$ or about $2 \%$ of the range of possible valve angles, however further study showed that lower $\epsilon$ 's are effective as well. This requirement was necessary due to the anomaly that was introduced (a delay) and is not required if one is seeking to detect parametric or additive faults such as those introduced to the valve and cooler. However, parametric and additive faults can still be detected with this excitation requirement in place, albiet with a longer detection time. Clearly, one has to know that a delay fault is of interest to put in place this excitation requirement. This places a requirement for limited a priori knowledge, but this fault has not traditionally been explored in automotive diagnosis literature and its detectability with the GSMMS method is significant.

Fig. 4.24 shows the CVs for the top level anomaly detection and Fig. 4.25 shows the mass flow subsystem ADs. The above anomaly detection and isolation procedure follows the methodology described in Chapter 3 with one notable modification. Since the EGR valve angle saturates $\left(0^{\circ} \leq \alpha \leq 90^{\circ}\right)$, the PI controller output has a saturation nonlinearity. Thus, the GSMMS output was saturated at the upper and lower limits of EGR valve travel. 


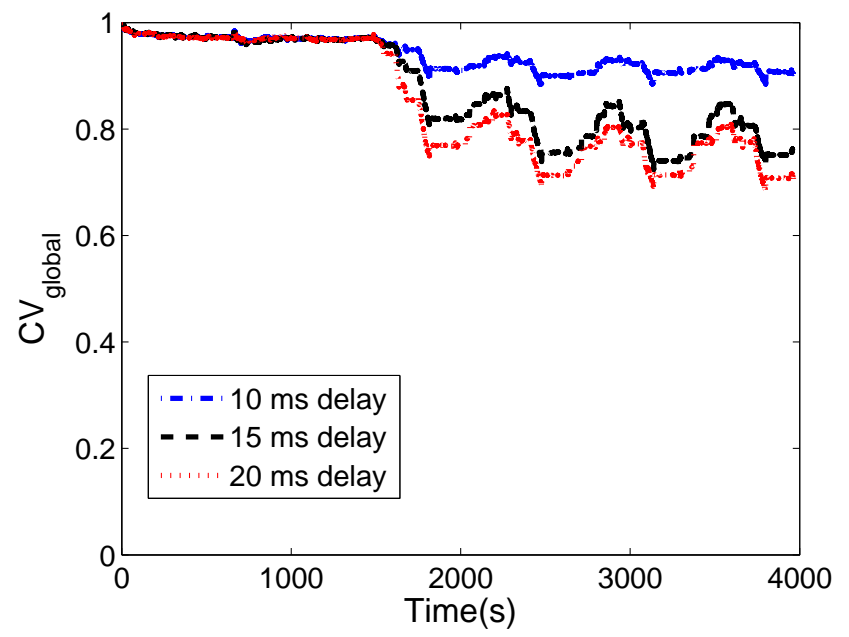

(a) $\mathrm{AD}_{\text {mflow }}$

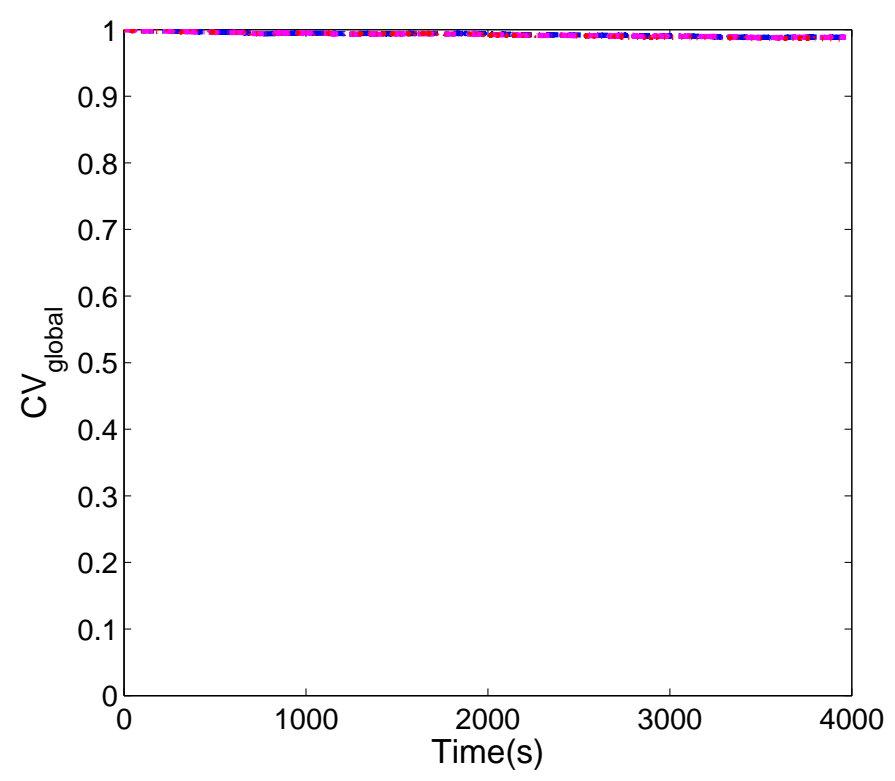

(b) $\mathrm{AD}_{\text {cooler }}$

Figure 4.24: $\mathrm{CV}_{\text {global }}$ for the top level anomaly detectors for a controller anomaly (time delay) introduced at 1322 seconds. 


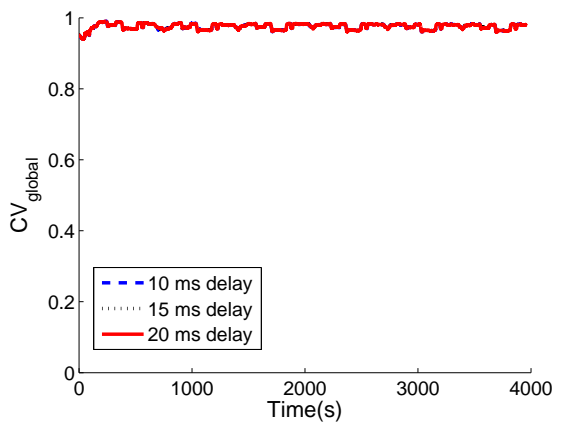

(a) $\mathrm{AD}_{2}$

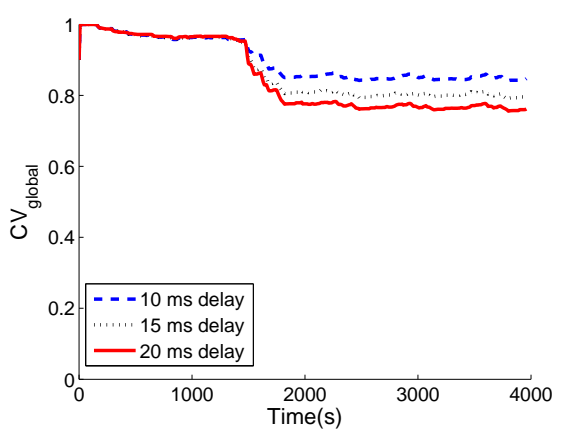

(c) $\mathrm{AD}_{4}$

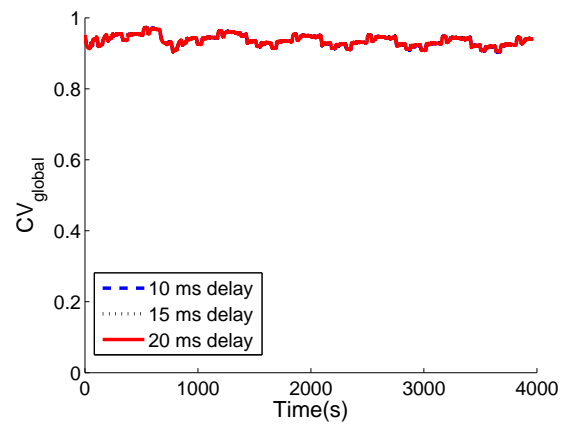

(b) $\mathrm{AD}_{3}$

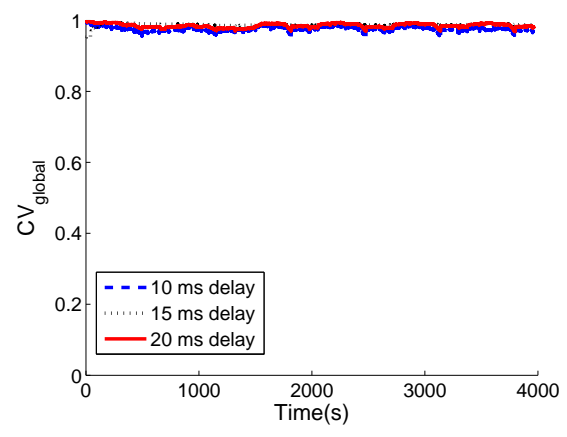

(d) $\mathrm{AD}_{5}$

Figure 4.25: Fault isolation using distributed anomaly detectors for a controller anomaly (time delay) introduced at 1322 seconds. The $\mathrm{CV}$ for $\mathrm{AD}_{4}$ drops while other CVs remain high. It can also be seen that the amount of $\mathrm{CV}$ reduction is proportional to the delay. 


\subsection{Fault Identification}

In this section, the GSMMS based fault identification scheme outlined in Section 3.4 is used to identify faults in the EGR subsystems. In the following, it is assumed that the anomaly is isolated to the appropriated subsystem and that a set of GSMMS models for faulty behavior are available. For the EGR application explored in this paper, a set of GSMMS models were trained using data emitted from a faulty system. Once an anomaly was detected, the CVs associated with each of these GSMMS models were monitored. The GSMMSs modeling system behavior in the presence of known faults as well as their regional CVs form a set of Diagnosers (Ds) for those known faults.

\subsubsection{EGR Valve Faults}

For the EGR Valve, diagnosers were contructed for valve faults 2 and 4. A simulation was conducted where the scaling factor, $\gamma$, was varied and the $\mathrm{CV}_{\text {global }}$ for each diagnoser were monitored. The results can be seen in Fig. 4.26. From this figure, it is clear that the global CVs for the diagnosers correctly identify the fault dynamics.

\subsubsection{EGR Cooler Faults}

For the EGR Cooler, diagnosers were contructed for valve faults 2 and 4 . A simulation similar to that of Section 4.5.1 was conducted where $\mathrm{CV}_{\text {global }}$ for each diagnoser was monitored. Instead of varying $\gamma, \beta$ was varied to simulate a set of cooler faults. The results can be seen in Fig. 4.27. The EGR cooler diagnosers cor- 


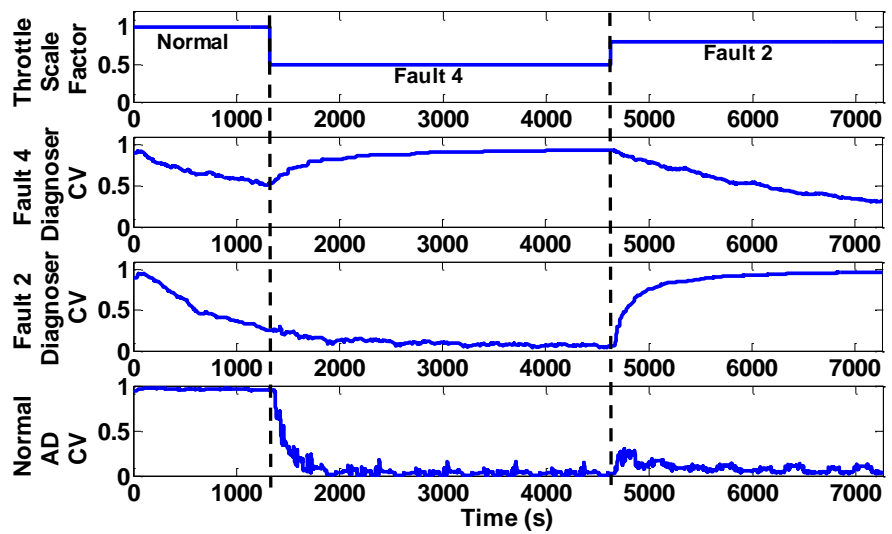

Figure 4.26: Valve scaling factor ( $\gamma$ in Eq. 4.4) and $\mathrm{CV}_{\text {global }}$ for valve diagnosers for faults $2 \& 4$. The scaling factor determines the current behavior of the EGR Valve, where $\gamma=0.8$ is Fault 2 and $\gamma=0.5$ is Fault 4.

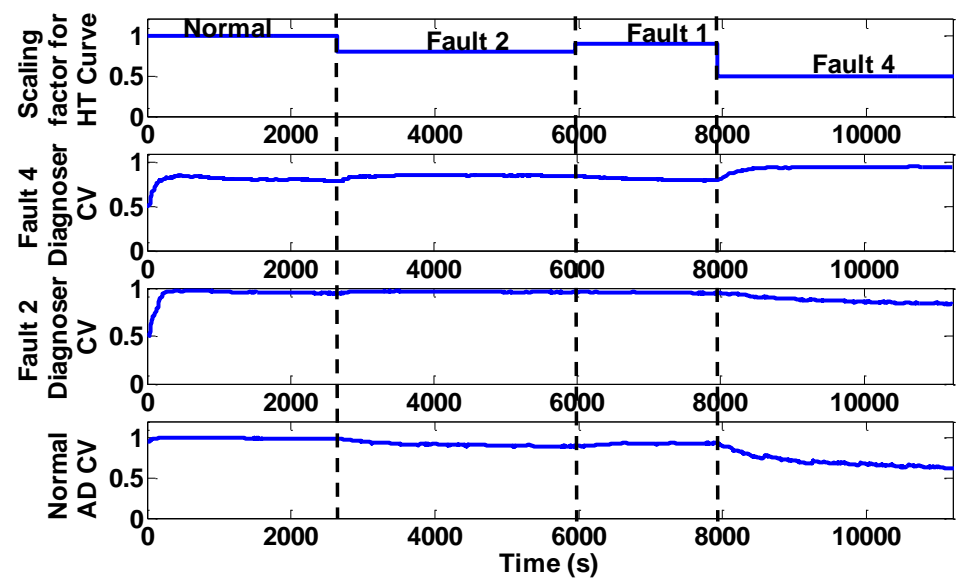

Figure 4.27: Cooler scaling factor and $\mathrm{CV}_{\text {global }}$ for cooler diagnosers for faults $2 \&$ 4. The scaling factor determines the current behavior of the EGR Cooler, where $\beta=0.8$ is Fault 2 and $\beta=0.5$ is Fault 4 .

rectly identify the faults, even though the sensitivity of the global CVs is relatively small and the values of the global CVs are relatively close together. However, the global CV does not necessarily indicate that the dynamics are represented well in 
all regions of the state space. As was stated previously, each GSMMS region can be sensitive (or insensitive) to different faults and the CVs of the regions can vary widely. In the case of the cooler anomaly detection, it has been shown that some local CVs are very sensitive while others are not. Since the drop of one regional $\mathrm{CV}$ indicates an anomaly, it may be more suitable to examine the local CV behavior rather than the (regional geometric) average.

Figure 4.28 shows representative regional $\mathrm{CVs}$ for the same diagnosers as Fig. 4.27. As in the case of the anomaly detection, some of the cooler CVs show large variations. Therefore, it may be beneficial to examine each regional $\mathrm{CV}$ during identification and require that all regional CVs must be high for the particular fault model to be selected, indicating that the model describes the system dynamics well in all regions.

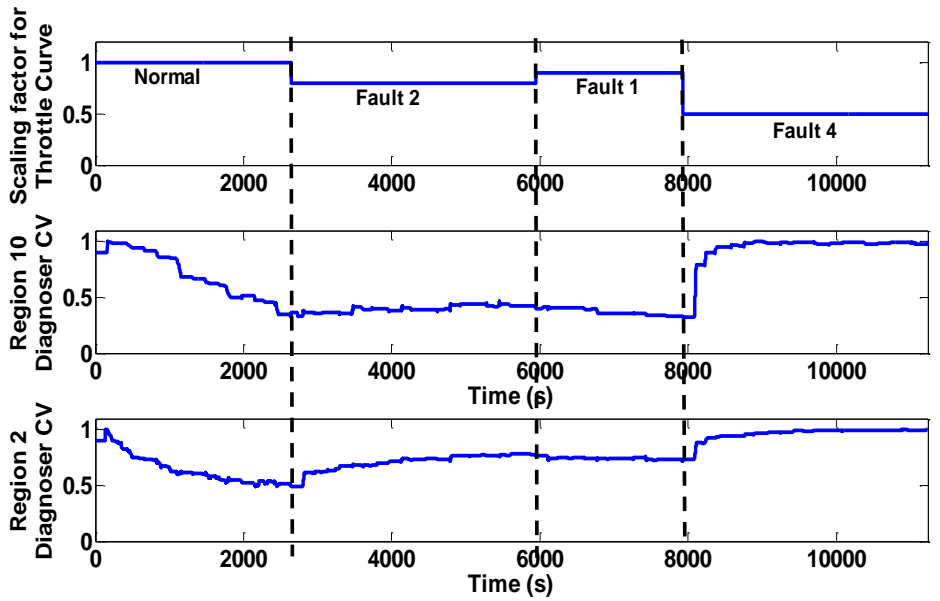

Figure 4.28: Regional CVs for Fault 4 diagnoser only. The regions shown here are clearly more sensitive than the global values in Fig. 4.27 and the regional CVs are high when Fault 4 is present, as expected. Note that the global CV for this diagnoser (4.27 is not nearly as sensitive as the local CVs shown. 


\subsubsection{PI Controller Faults}

A diagnoser for the $20 \mathrm{~ms}$ controller delay was constructed for the PI contrtoller. This diagnoser was monitored over time for various delay faults and the results can be seen in Fig. $4.29^{3}$. From the figure, it can be seen that during the period of time where the 20 ms delay is present (from 8000s-13000s) has the highest $\mathrm{CV}$, as expected. However, the diagnoser $\mathrm{CV}$ remains high when the the $10 \mathrm{~ms}$ delay is present and thus the 10 and $15 \mathrm{~ms}$ faults cannot be practically differentiated using this diagnoser. It should be noted, however, that this single-region model is simple to estimate using a linear least squares estimator. In the case of the PI controller, it may be simpler to re-estimate the model online when an anomaly is detected.

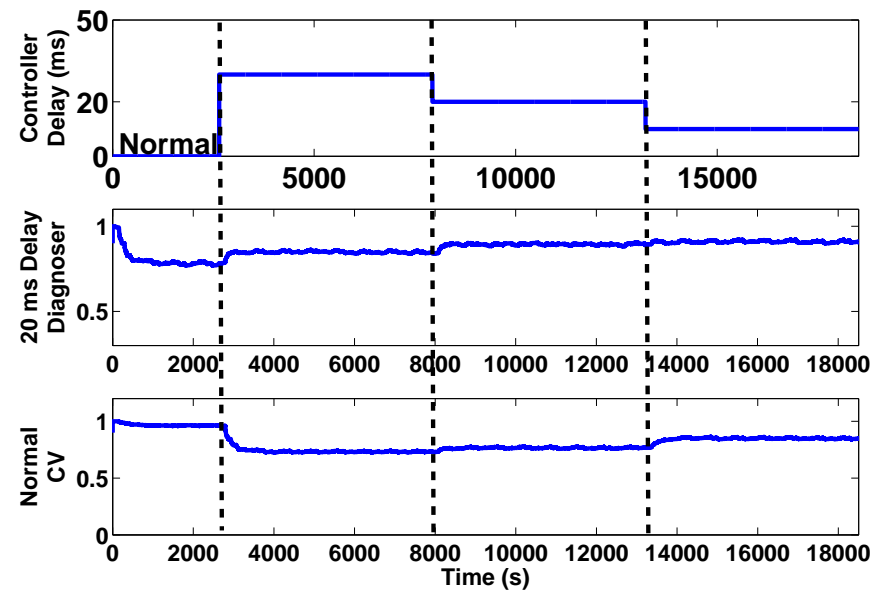

Figure 4.29: $20 \mathrm{~ms}$ diagnoser $\mathrm{CV}$ for the PI controller in the presence of various delays. The $\mathrm{CV}$ during the $20 \mathrm{~ms}$ fault period is different from the $30 \mathrm{~ms}$ and normal periods, but the $10 \mathrm{~ms}$ fault period maintains a high $\mathrm{CV}$.

\footnotetext{
${ }^{3}$ Since there is only one region for the PI controller, there are no regional CVs
} 


\section{Chapter 5}

\section{Conclusions and Future Work}

\subsection{Conclusions}

In this thesis a novel Fault Detection and Diagnosis (FDD) method was designed for a Diesel Engine Exhaust Gas Recirculation (EGR) system. This system consists of a valve, cooler and controller that constitute a set of dynamic, nonlinear interacting subsystems. The methodology in this thesis addresses four major issues present in automotive air path anomaly detection:

- Current fault detection method in automotive systems utilize limit checks that must be set widely due to system noise and operating point changes.

- The need for extensive a priori knowledge to isolate faults by propagating anticipated faults through a series of interacting dynamic systems.

- Complications in detection involving highly nonlinear interacting dynamic systems.

- Lack of a framework for estimation of new (faulty) dynamics after isolation.

To address these problems, a model-based approach was utilized. Model-based approaches use a system model (physical, data-driven, signal model, etc.) to describe 
input-output relationships during normal operation. Such a methodology stand in contrast with current "limit check" methods, which do not utilize input or process information to condition fault detection decisions. By conditioning the expected output on the input, a set of residuals (modeling errors) can be generated for normal system operation. By monitoring the residual signals, faults can be detected as statistically significant (overlap of residual distributions) departures from the normal residuals.

While model-based approaches have significant benefits, the success is heavily dependent on the quality of the model. In this thesis, the recently introduced Growing Structure Multiple Model System (GSMMS) was used to construct models of the normal behavior of the EGR system and constituent subsystems. Using data emitted from a system, the GSMMS partitioned the state-space into regions of similar dynamic behavior and fit linear Autoregressive Exogenous models [18] within each region. The result is a "tiling" of the nonlinear system model where regions with highly nonlinear behavior contain more "tiles" to obtain a better approximation of the global dynamics.

Using the GSMMS models as a characterization of normal behavior, anomaly detectors (ADs) for the mass flow and cooler subsystems were constructed whose task was to detect departures away from normal behavior. Each AD consists of a GSMMS model that ran in parallel with the actual system. The respective outputs were then subtracted, generating modeling residuals that were used to characterize the system behavior. Under ideal conditions, the modeling residuals would be zero for normal behavior and greater than zero when a anomaly (fault) is present. 
An anomaly was then signaled when the statistical characteristics of the current modeling residuals departed significantly from the modeling residuals observed during normal operation. This was accomplished by estimating the probability density function (PDF) of the normal modeling residuals using Gaussian mixture models [52]. PDFs of the most recently observed GSMMS residuals were continuously updated and compared to the normal residuals by computing a normalized area of intersection between the two PDFs. Following [44], this intersection was referred to as the performance Confidence Value (CV). Thus, the CV would be one for normal operation and less than one for faulty operation. For simplicity of monitoring, regional CVs were combined into a global $\mathrm{CV}$ representing an indicator of overall system health. Since only normal data is used in training the GSMMS, drops in CVs were used to detect faults without knowledge of the fault or utilization of faulty data. One important observation was the behavior of the global CV compaired to that of the local CVs. Mainly, one could find a local CV that was sensitive to that fault whereas the global CVs were not sensitive to some less severe faults. This happened because the calculation of the global $\mathrm{CV}$ averaged the sensitive local region with regions were system behavior appeared normal. For this reason, it is clearly preferable to monitor the individual regional CVs as indicators of system health.

Once an anomaly was detected, fault isolation was conducted by distributing anomaly detectors throughout the constituent subsystems. By monitoring this set of CVs, the anomaly was isolated to the smallest possible granularity without prior knowledge or training for the underlying fault (precedent-free). In this thesis, anomalies in the EGR valve, cooler and PI controller were isolated in this 
precedent-free manner.

Furthermore, it was shown how the GSMMS based methodology could be used to detect previously encountered faults. Once a fault was detected and isolated, GSMMS models that were constructed using faulty data were used to generate a new set of modeling residuals. In the same manner as the ADs, CVs were constructed and monitored. However, in this situation the $\mathrm{CV}$ represented the confidence that the particular fault model accurately described the behavior of the current system. If none of these "diagnoser" CVs were high, the fault is classified as unprecedented and a new GSMMS can be trained to recognize this fault in the future. In the EGR system, this methodology was used to identify a subset of the anomalies introduced in the EGR subsystems. While some of the faults were indistinguishable using the global CVs, the local CVs were more sensitive and could distinguish between faults. This observation reinforces the idea that one needs to base decisions on a more intelligent fusion of local CVs to decide which behavior mode is present in the current system operation.

\subsection{Future Work}

The focus of this thesis was on demonstrating the feasibility of precedent free fault detection and isolation in automotive systems through an application to a diesel engine EGR system. However, several major issues remain in the use of GSMMS based diagnostics. In this section, future research tasks will be discussed based on the author's experience during this research. 


\subsubsection{Application to a Real Engine and Benchmarking}

In this thesis a high-fidelity engine simulator was utilized to simulate the engine. While this simulator accounts for many complexities of the engine dynamics, real engines will have significantly more uncertainty than was simulated in this thesis. Sensor noise, heat losses, and non-ideal air and fuel flows are some of the factors that are not accounted for in simulations. Therefore, an area of future work will be to acquire real engine data to test the GSMMS based methodology in an actual engine application. Furthermore, it would be advantageous to test the methodology using fault scenarios that other researchers have employed in order to benchmark the GSMMS methodology. In Chapter 2 numerous model-based method were discussed and the faults introduced in these experiments could server as a starting point. For example, one could introduce leaks into the air path of varying diameters as was done in $[10,13]$. Whatever experiments are conducted, validation of the GSMMS capabilities to handle the noisy, uncertain environment of a real engine will be beneficial.

\subsubsection{Controller Reconfiguration}

With increasingly demanding performance and robustness requirements, Fault Tolerant Control Systems (FTCS) have received much attention in literature. An attractive class of such controllers are the so-called active FTCS where the controller is able to reconfigure to maintain stability and acceptable performance [60]. An area of future work will be to use the GSMMS models to derive a control reconfiguration law that enables such structures. 
While FTCS methods have recently been employed for nonlinear systems $[61,62]$ the majority of the literature concentrates on utilizing linear models. Among the linear methods, some popular choices are the Pseudo-Inverse Method (PIM) [63-65] and Eigenstructure Assignment (EA) [66] methods, which essentially seek to match the eigenvalues of a reference model with the current (faulty) one. Since the GSMMS FDD procedure produces models that are locally affine, there exists a potential to employ these methods in each region of the GSMMS using the normal model as the desired behavior and the fault model as the current behavior.

However, in such controller reconfiguration, a characterization of stability could be necessary to ensure that the resulting closed loop system is stable. While the stability of the GSMMS model is an open question, methods have been developed to asses the stability of local piecewise models $[46,67]$. Thus as a subtask of the reconfiguration work, the stability of the GSMMS models will be assessed and be utilized to characterize the resulting control laws. 


\section{Bibliography}

[1] V. Krishnaswami, G. Luh, and G. Rizzoni. Nonlinear parity equation based residual generation for diagnosis of automotive engine faults. Control Engineering Practice, 3(10):1385-1392, October 1995.

[2] M.Zheng, G.T. Reader, and J.G. Hawley. Diesel engine exhaust gas recirculationa review on advanced and novel concepts. Energy Conversion and Management, 45(6):883-900, 2004.

[3] Final regulation orders for OBD II and emission warranty regulations, November 2007.

[4] J. J. Gertler. Fault Detection and Diagnosis in Engineering Systems. Marcel Dekker, 1998.

[5] F. Kimmich, A. Schwarte, and R. Isermann. Fault detection for modern Diesel engines using signal- and process model-based methods. Control Engineering Practice, 13(2):189-203, February 2005.

[6] G. Rizzoni and P.S. Min. Detection of sensor failures in automotive engines. Vehicular Technology, IEEE Transactions on, 40(2):487-500, May 1991.

[7] J. Gertler. Model-based on-board fault detection and diagnosis for automotive engines. Control Engineering Practice, 1(1):3-17, 1993. 
[8] R.J. Patton and C.J. Lopez-Toribio. Soft computing approaches to fault diagnosis for dynamic systems: a survey. In Proceedings of the 4 th IFAC Symposium SAFEPROCESS 'O0, pages 298-311. Elsevier, 2000.

[9] R. Conatser, J. Wagner, S. Ganta, and I. Walker. Diagnosis of automotive electronic throttle control systems. Control Engineering Practice, 12(1):2330, January 2004.

[10] J. Mohammadpour, K.M. Grigoriadis, M.A. Franchek, and B.J. Zwissler. Realtime diagnostics in the EGR system of diesel engines. In American Control Conference, 2008, pages 1002-1007, June 2008.

[11] S.X. Ding, N. Weinhold, P. Zhang, E.L. Ding, T. Jeinsch, and M. Schultalbers. Integration of fdi functional units into embedded tracking control loops and its application to fdi in engine control systems. pages 1299-1304, Aug. 2005.

[12] R. Cecarelli, C. C. De Wit, P. Moulin, and A. Sciarretta. Model-based Adaptive Observers for Intake Leakage Detection in Diesel Engins. American Control Conference, IEEE ACC'09, 2009.

[13] M. Nyberg and T. Stutte. Model based diagnosis of the air path of an automotive diesel engine. Control Engineering Practice, 12(5):513 - 525, 2004. Fuzzy System Applications in Control.

[14] Y.W. Kim, G. Rizzoni, and V. Utkin. Automotive engine diagnosis and control via nonlinear estimation. Control Systems Magazine, IEEE, 18(5):84-99, Oct 1998. 
[15] D. Antory. Application of a data-driven monitoring technique to diagnose air leaks in an automotive diesel engine: A case study. Mechanical Systems and Signal Processing, 21(2):795 - 808, 2007.

[16] J. Edward Jackson. A User's Guide to Principal Components. John Wiley \& Sons, Inc., 1991.

[17] S. A. Billings, M. B. Fadzil, J. L. Sulley, and P. M. Johnson. Identification of a non-linear difference equation model of an industrial diesel generator. Mechanical Systems and Signal Processing, 2(1):59 - 76, 1988.

[18] E. Ikonen and K. Najim. Advanced Process Identification and Control. Marcel Dekker, 2002.

[19] M. A. Franchek, P. J. Buehler, and I. Makki. Intake air path diagnostics for internal combustion engines. Journal of Dynamic Systems, Measurement, and Control, 129(1):32-40, 2007.

[20] D. Capriglione, C. Liguori, C. Pianese, and A. Pietrosanto. On-line sensor fault detection, isolation, and accommodation in automotive engines. In Instrumentation and Measurement Technology Conference, 2002. IMTC/2002. Proceedings of the 19th IEEE, volume 2, pages 1711-1716 vol.2, 2002.

[21] D.W. Dong, J.J. Hopfield, and K.P. Unnikrishnan. Neural networks for engine fault diagnostics. pages 636-644, Sep 1997.

[22] X. Wang, N. McDowell, U. Kruger, G. McCullough, and G.W. Irwin. Semiphysical neural network model in detecting engine transient faults using the 
local approach. In The International Federation of Automatic Control Proceedings of the 17th World Congress, pages 7086-7090, Seoul, Korea, July 2008.

[23] J.A.F. Vinsonneau, D.N. Shields, P.J. King, and K.J. Burnham. Improved si engine modelling techniques with application to fault detection. In Control Applications, 2002. Proceedings of the 2002 International Conference on, volume 2, pages 719-724 vol.2, 2002.

[24] J.J. Gertler, M. Costin, X. Fang, Z. Kowalczuk, M. Kunwer, and R. Monajemy. Model based diagnosis for automotive engines-algorithm development and testing on a production vehicle. Control Systems Technology, IEEE Transactions on, 3(1):61-69, Mar 1995.

[25] A. Soliman, G. Rizzoni, and Y. W. Kim. Diagnosis of an automotive emission control system using fuzzy inference. Control Engineering Practice, 7(2):209 $-216,1999$.

[26] R. Isermann. Model-based fault-detection and diagnosis - status and applications. Annual Reviews in Control, 29(1):71 - 85, 2005.

[27] Y.L. Murphey, J.A. Crossman, ZhiHang Chen, and J. Cardillo. Automotive fault diagnosis - part ii: a distributed agent diagnostic system. Vehicular Technology, IEEE Transactions on, 52(4):1076-1098, July 2003.

[28] J. J. Gertler, M. Costin, X. Fang, Z. Kowalczuk, M. Kunwer, and R. Monajemy. Model based diagnosis for automotive engines-algorithm development 
and testing on a production vehicle. IEEE Transactions on Control Systems Technology, 3(1):61-69, 1995.

[29] P. Struss and C. Price. Model-based systems in the automotive industry. AI Mag., 24(4):17-34, 2004.

[30] Jianbo Liu. Autonomous Anomaly Detection and Fault Diagnosis. PhD thesis, University of Michigan, 2007.

[31] M. Nyberg. Model-based diagnosis of an automotive engine using several types of fault models. Control Systems Technology, IEEE Transactions on, 10(5):679-689, Sep 2002.

[32] V. Kecman. Learning and Soft Computing. The MIT Press, 2001.

[33] J.C. Principe, Ludong Wang, and M.A. Motter. Local dynamic modeling with self-organizing maps and applications to nonlinear system identification and control. Proceedings of the IEEE, 86(11):2240-2258, Nov 1998.

[34] T. Takagi and M. Sugeno. Fuzzy identification of systems and its applications to modeling and control. IEEE Transa. on Systems, Man and Cybernetics, 15(1):116-132, 1985.

[35] F.K. Lewis and V.L. Syrmos. Optimal Control. John Wiley \& Sons, Inc., 2nd edition, 1995.

[36] S.G. Tzafestas and K.C. Zikidis. NeuroFAST: on-line neuro-fuzzy ARTbased structure and parameter learning TSK model. IEEE Trans. on Systems, Man, and Cybernetics, Part B, 31(5):797-802, Oct 2001. 
[37] T.A. Johansen and B.A. Foss. Identification of non-linear system structure and parameters using regime decomposition. Automatica, 31(2):321-326, 1995.

[38] T. Kohonen. Self-organized formation of topologically correct feature maps, pages 509-521. MIT Press, Cambridge, MA, 1988.

[39] G.A. Barreto and A.F.R. Araujo. Identification and control of dynamical systems using the self-organizing map. Neural Networks, IEEE Transactions on, 15(5):1244-1259, Sept. 2004.

[40] M. Ge, M.S. Chin, and Q.G. Wang. An extended self-organizing map for nonlinear system identification. Decision and Control, 1999. Proceedings of the 38th IEEE Conference on, 1:1065-1070, 1999.

[41] B.Fritzke. A growing neural gas network learns topologies. In Neural Information Processing Systems, pages 625-632, 1994.

[42] B. Fritzke. Growing cell structures - a self-organizing network for unsupervised and supervised learning. Neural Networks, 7(9):1441-1460, 1994.

[43] D. Alahakoon, S.K. Halgamuge, and B. Srinivasan. Dynamic self-organizing maps with controlled growth for knowledge discovery. Neural Networks, IEEE Transactions on, 11(3):601-614, May 2000.

[44] J. Liu, D. Djurdjanovic, K. Marko, and J. Ni. Growing structure multiple model systems for anomaly detection and fault diagnosis. Journal of Dynamic Systems, Measurement, and Control, 131(5):051001, 2009. 
[45] J. Liu and D. Djurdjanovic. Topology Preservation and Cooperative Learning in Identification of Multiple Model Systems. Neural Networks, IEEE Transactions on, 19(12):2065-2072, Dec. 2008.

[46] D. Mignone, G. Ferrari-Trecate, and M. Morari. Stability and stabilization of piecewise affine and hybrid systems: an LMI approach. Decision and Control, 2000. Proceedings of the 39th IEEE Conference on, 1:504-509, 2000.

[47] D. Djurdjanovic, J. Liu, K. Marko, and J. Ni. Immune Systems Inspired Approach to Anomaly Detection and Fault Diagnosis for Engines. Neural Networks, 2007. IJCNN 2007. International Joint Conference on, pages 13751382, August, 2007 12-17 Aug. 2007.

[48] J. Liu, P. Sun, D. Djurdjanovic, K. Marko, and J. Ni. Growing Structure Multiple Model System Based Anomaly Detection for Crankshaft Monitoring. Advances in Neural Networks - ISNN 2006, 3973:396-405, 2006.

[49] R. Sedgewick. Algorithms in C++. Part 5, Grapical Algorithms. AddisonWesley, London, 1995.

[50] T. Kohonen. Self Organizing Maps. Springer Series in Information Sciences. Springer-Verlag, 1995.

[51] L. Ljung. System Identification: Theory for the User. Prentice-Hall, 1987.

[52] G. McLachlan and D. Peel. Finite Mixture Models. John Wiley \& Sons, Inc., 2000. 
[53] Z. Zivkovic and F. van der Heijden. Recursive Unsupervised Learning of Finite Mixture Models. IEEE Trans. on Pattern Analysis and Machine Intelligence, 26(5):651-656, 2004.

[54] TESIS DYNAware. $\quad$ en-DYNA ${ }^{\circledR}$ THEMOS ${ }^{\circledR} 2.0$ Block Reference Manual, June 2006.

[55] Sudhakar M. Pandit and Shien-Ming Wu. Time Series and System Analysis with Applications. Krieger, 1983.

[56] Peter D. Grnwald, In Jae Myung, and Mark A. Pitt, editors. Advances in Minimum Description Length Theory and Applications. The MIT Press, 2005.

[57] L. Jiang, E. Latronico, and J. Ni. A Novel Method for Input Selection for the Modeling of Nonlinear Dynamic Systems. Proceedings of ASME Dynamic Systems and Control Conference, October 2008. Paper no. DSCC2008-2217.

[58] Katsuhiko Ogata. System Dynamics. Prentice Hall, 2004.

[59] J. Liu, D. Djurdjanovic, K. Marko, and J. Ni. A divide and conquer approach to anomaly detection, localization and diagnosis. Mechanical Systems and Signal Processing, 23(8):2488 - 2499, 2009.

[60] Y. Zhang and J. Jiang. Bibliographical review on reconfigurable fault-tolerant control systems. Annual Reviews in Control, 32(2):229 - 252, 2008.

[61] Y. Diao and K. M. Passino. Intelligent fault-tolerant control using adaptive and learning methods. Control Engineering Practice, 10(8):801 - 817, 2002. 
[62] M.M. Polycarpou. Fault accommodation of a class of multivariable nonlinear dynamical systems using a learning approach. Automatic Control, IEEE Transactions on, 46(5):736-742, May 2001.

[63] Z. Gao and P.J. Antsaklis. On the stability of the pseudo-inverse method for reconfigurable control systems. In Aerospace and Electronics Conference, 1989. NAECON 1989., Proceedings of the IEEE 1989 National, pages $333-$ 337 vol.1, May 1989.

[64] M. Staroswiecki. Fault tolerant control using an admissible model matching approach. pages 2421-2426, Dec. 2005.

[65] M Staroswiecki. Fault tolerant control: The pseudo-inverse method revisited. Proceedings of the 16th IFAC World congress, 16:418-423, 2005.

[66] Y. Zhang and J. Jiang. Integrated active fault-tolerant control using imm approach. Aerospace and Electronic Systems, IEEE Transactions on, 37(4):12211235, Oct 2001.

[67] M. Johansson and A. Rantzer. Computation of piecewise quadratic Lyapunov functions for hybrid systems. Automatic Control, IEEE Transactions on, 43(4):555-559, Apr 1998. 


\section{Vita}

Michael Cholette was born in Rochester, Michigan, USA. After growing in Clarkston, Michigan he attended The University of Michigan-Ann Arbor where he studied Mechanical Engineering. He received a Bachelor of Science in Mechanical Engineering from the University of Michigan in 2007 and spent the fall of 2005 and summer 2006 at Caterpillar. After spending the summer of 2007 at Ford Motor Company, he enrolled in graduate school at The University of Texas at Austin.

Permanent address: 5221 Old Cove Rd.

Clarkston, MI 48346

This thesis was typeset with $\mathrm{LT}_{\mathrm{E}} \mathrm{X}^{\dagger}$ by the author.

\footnotetext{
${ }^{\dagger} \mathrm{HT}_{\mathrm{E}} \mathrm{X}$ is a document preparation system developed by Leslie Lamport as a special version of Donald Knuth's TEX Program.
} 